\title{
Geochemical characterization of the largest upland lake of the Brazilian Amazonia: Impact of provenance and processes
}

\author{
Prafulla Kumar Sahoo ${ }^{\text {a, * }}$, José Tasso Felix Guimarães ${ }^{\text {a }}$, \\ Pedro Walfir Martins Souza-Filho ${ }^{\text {a, b }}$, Marcio Sousa da Silva ${ }^{a}$, Wilson Nascimento Júnior a , \\ Mike A. Powell ${ }^{c}$, Luiza Santos Reis ${ }^{d}$, Luiz Carlos Ruiz Pessenda ${ }^{\mathrm{d}}$, \\ Tarcísio Magevski Rodrigues ${ }^{e}$, Delmo Fonseca da Silva ${ }^{e}$, Vladimir Eliodoro Costa ${ }^{f}$ \\ a Instituto Tecnológico Vale, Rua Boaventura da Silva 955, Nazaré 66055-090 Belém, Pará, Brazil \\ b Universidade Federal do Pará, Instituto de Geociências, Programa de Pós-graduação em Geologia e Geoquímica. Av. Augusto Correa 1, Guamá, 66075-110, \\ Belém, Pará, Brazil \\ ${ }^{\mathrm{c}}$ Independent Geologist \& Pres., Geocon Environmental Consulting, London, ON, N6G3H9, Canada \\ ${ }^{d}$ Centro de Energia Nuclear na Agricultura, Laboratório C-14, Av. Centenário, 303, São Dimas, 13400-970, Piracicaba, São Paulo, Brazil

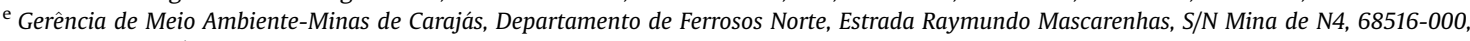 \\ Parauapebas, Pará, Brazil \\ ${ }^{\mathrm{f}}$ Centro de Isótopos Estáveis, Instituto de Biociências, Universidade Estadual Paulista, Distrito de Rubião Junior s/n, 18618-970, Botucatu, SP, Brazil
}

\section{A R T I C L E I N F O}

\section{Article history:}

Received 29 July 2017

Received in revised form

24 October 2017

Accepted 24 October 2017

Available online 26 October 2017

\section{Keywords:}

Sediment geochemistry

Upland lakes

Provenance

Catchment lithology

Amazon region

\begin{abstract}
A B S T R A C T
Lake Três Irmãs (LTI), the largest upland lake in the Brazilian Amazonia, located in Serra dos Carajás, was characterized using multi-elemental and isotope geochemistry $\left(\delta^{13} \mathrm{C}\right.$ and $\left.\delta^{15} \mathrm{~N}\right)$ to understand the significance of organic and inorganic sources, weathering and sedimentary processes on the distribution of elements in lake bottom (surficial) sediments. Carbon and nitrogen isotopes from sedimentary organic matter suggest C3 terrestrial plants (forests > canga vegetation), macrophytes and freshwater DOC as the main sources. Sediments are depleted in most of the major oxides (except $\mathrm{Fe}_{2} \mathrm{O}_{3}$ and $\mathrm{P}_{2} \mathrm{O}_{5}$ ) when compared to upper continental crust (UCC) and their spatial distribution is highly influenced by catchment lithology. Principal Component Analysis revealed that most of the trace elements ( $\mathrm{Ba}, \mathrm{Sr}, \mathrm{Rb}, \mathrm{Sc}, \mathrm{Th}, \mathrm{U}, \mathrm{Zr}, \mathrm{Hf}, \mathrm{Nb}, \mathrm{Y}, \mathrm{V}, \mathrm{Cr}, \mathrm{Ga}, \mathrm{Co}, \mathrm{Ni}$ ) and REEs are closely correlated with $\mathrm{Al}$ and $\mathrm{Ti}$ (PC1; Group-1), so their redistribution is less influenced by post-depositional process. This is due to their relative immobility and being hosted by Al-bearing minerals during laterization. High Chemical Index of Alteration (CIA), Mafic Index of Alteration (MIA) and Index of Laterization (IOL) values indicate intense chemical weathering at source areas, but the weathering transformation was better quantified by IOL. A-CN-K plot along with elemental ratios $(\mathrm{Al} / \mathrm{K}, \mathrm{Ti} / \mathrm{K}, \mathrm{Ti} / \mathrm{Zr}, \mathrm{La} / \mathrm{Al}, \mathrm{Cr} / \mathrm{Th}, \mathrm{Co} / \mathrm{Th}, \mathrm{La} / \mathrm{Sm}, \mathrm{La} / \mathrm{Gd}, \mathrm{Zr} /$ $\mathrm{Y}$, and $\left.\mathrm{Eu} / \mathrm{Eu}^{*}\right)$ as well as chondrite-normalized REE patterns show that the detritic sediments are mainly sourced from ferruginous laterites and soils in the catchment, which may have characteristics similar to mafic rocks.
\end{abstract}

(c) 2017 Elsevier Ltd. All rights reserved.

\section{Introduction}

The Brazilian Amazon has the largest tropical forest in the world and includes several floodplains and upland lakes, which are a main source of information for understanding the paleoclimatic conditions of the Amazon (Latrubesse, 2012). Três Irmâs lake is the

\footnotetext{
* Corresponding author. Instituto Tecnológico Vale, Belém, Brazil.

E-mail addresses: prafulla.sahoo@itv.org, prafulla.iitkgp@gmail.com (P.K. Sahoo)
}

largest upland lake (with surface area of $\sim 0.5 \mathrm{~km}^{2}$; Silva et al., submitted) in Brazilian Amazonia, situated in the Carajás mineral province. Contrary to fluvial floodplain lakes, upland lakes are hydrologically restricted and in this case formed over lateritic crusts by structural and weathering processes (Maurity and Kotschoubey, 1995). In this region, lakes are situated at altitudes of around $700 \mathrm{~m}$ and are highly influenced by the natural characteristics of their catchments (Sahoo et al., 2015). Therefore, lake sediments of this region have been the focus of intense studies in recent years, especially related to paleoclimate studies based on palynology and 
mineralogy (Soubies et al., 1991; Sifeddine et al., 2001; Costa et al., 2005; Hermanowski et al., 2012; Guimarães et al., 2016a, in press), and a few geochemical (Sahoo et al., 2015, 2016) and sedimentological studies (Souza-Filho et al., 2016).

The chemical (organic and inorganic) and physical character of lake bottom sediments is affected by various factors such as hydraulic sorting, weathering rates, erosion, transport pathways, grain-size distribution, source rock composition and the tectonic setting of the basin (Das and Haake, 2003; Jin et al., 2006; Vogel et al., 2010; Sahoo et al., 2015; Babeesh et al., 2017). Postdepositional diagenetic changes, including precipitation of authigenic Fe-oxyhydroxide (e.g. goetheite, jarosite, and magnetite), sulfides (e.g. pyrite), carbonates (e.g. siderite, calcite and aragonite) and phosphates (e.g. vivianite), may also control redistribution of trace elements in lake sediments (Audry et al., 2010; Jelinowska et al., 2000; Sahoo et al., 2015). Sediment elemental ratios can be altered during weathering (Taylor and McLennan, 1985; Potter et al., 2005) and post-depositional modification of clay minerals (Xiao et al., 2010) and/or diagenesis (Milodowski and Zalasiewiez, 1991). However, as long as the bulk chemical composition of the sediment is not significantly altered, the geochemical signatures retained within sediments can be used as an essential tool to reconstruct distribution patterns as well as weathering and provenance histories (Taylor and McLennan, 1985). Isotopic concentrations of $\delta^{13} \mathrm{C}$ and $\delta^{15}$ along with $\mathrm{C} / \mathrm{N}$ ratio are being widely used to understand the source of organic matter and the relative contribution of vascular and non-vascular plants to lake sediments (Meyers, 1994; Wei et al., 2010; Smith et al., 2012; Sahoo et al., 2015). The effect of diagenesis on $\delta^{13} \mathrm{C}$ and $\delta^{15} \mathrm{~N}$ signals of organic matter preserved in sediments may still begin in the water column by microorganism activity, which may influence in the primary isotopic values (Chen et al., 2008). However, several authors have indicated that once buried, the organic matter preserved in sediments will just present minor changes in $\delta^{13} \mathrm{C}$, allowing its use as a geochemical proxy (Thornton and McManus, 1994; Middelburg and Nieuwenhuize, 1998; Sahoo et al., 2015). Source rock contributions are determined using concentrations and elemental ratios including $\mathrm{Al}, \mathrm{Ti}, \mathrm{Sc}, \mathrm{Zr}, \mathrm{Hf}, \mathrm{Ng}, \mathrm{Y}, \mathrm{Cr}$ and REE due to their low mobility during weathering, transport and diagenesis (Taylor and McLennan, 1985; Wang et al., 2014). Geochemical signatures of clastic lake sediments have also been used to study the inherited relationships between weathering product and parent rocks, which can help in understanding the evolution of the lake as well as environmental variability in its terrestrial realms (Vogel et al., 2010; Wennrich et al., 2013; Sahoo et al., 2016). During chemical weathering, elements with high mobility are leached from source rocks while less mobile elements such as Fe and $\mathrm{Al}$ are remained in the residue. This is commonly estimated by using the Chemical index of Alteration (CIA) accompanying A-CN-K diagram, and Chemical Index of Weathering (CIW) based on the major element of detrital sediments (Nesbitt et al., 1996; Fedo et al., 1995). Recently, Babechuk et al. (2014) proposed a new weathering index, the Mafic Index of Alteration (MIA), which is an extension of CIA, allowing the mafic mineral component of rock weathering to be quantified.

Multi-proxy geochemical approaches can provide important information for understanding modern sedimentary processes and source-catchment interactions in any given geological setting and how they control elemental distribution in surficial lake sediments. However, these studies are largely limited, as is the case for Três Irmâs Lake. In the present work, concentrations of major, trace and REEs, isotopes of $\delta^{13} \mathrm{C}$ and $\delta^{15} \mathrm{~N}$, and $\mathrm{C} / \mathrm{N}$ ratios were used as proxies to determine occurrence and distribution of organic and inorganic sediments as related to provenance and modern sedimentary processes.

\section{Study area, geology and vegetation patterns}

The study area is a narrow NW-SE tending plateau that rises above a forested landscape at an altitude of around $730 \mathrm{~m}$, located in the Serra Sul de Carajás, southeastern Amazonia (Fig. 1). This region represents the major Archean Province of the Amazonian Craton (Macambira and Lafon, 1995; Rämö et al., 2002), which includes basaltic andesite, shoshonites and rhyolites intruded by quartz diorites of the Parauapebas Formation (Gibbs et al., 1986; Teixeira and Eggler, 1994) and banded iron formations (BIFs) of the Carajás Formation (Olszewski et al., 1989). Large Fe-ore deposits are associated with this latter formation. In tropical climates intense weathering leads to formation of lateritic crusts, which produce detrital and iron ore duricrusts (Golder, 2010, Fig. 1A). The surface of the plateau contains several lakes in a closed catchment system, isolated from fluvial influence, which were formed by weathering processes during laterization of the underlying crusts (Maurity and Kotschoubey, 1995). These lakes may be classified as active or inactive (filled) depending on lake levels (e.g. Sahoo et al., 2016; Guimarães et al., 2016b). The latter are easily identified from satellite images by the level of macrophytes and palms established in the central basins of the lakes, which form upland swamps.

Três Irmãs Lake has surface area, maximum depth and volume of around $0.5 \mathrm{~km}^{2}, 15 \mathrm{~m}$ and 2,325,200 $\mathrm{m}^{3}$, respectively (Silva et al., submitted). The lake is formed by the coalescence of three lakes (Três Irmãs Lake (LTI) 1 to 3; Fig. 1B). LTI1 is the smallest with an area of $0.07 \mathrm{~km}^{2}$, LTI3 is the largest and deepest with area and maximum depth of $\sim 0.25 \mathrm{~km}^{2}$ and $15 \mathrm{~m}$, respectively, and LTI2 is the shallowest with maximum and mean depth of $\sim 3$ and $1 \mathrm{~m}$, respectively (Silva et al., submitted). LTI2 and LTI3 can be hydrologically connected during wetter seasons. Ferruginous-detritic and aluminous-ferruginous crusts underlie most of the catchment basin (Fig. 1A), while its western borders are related to the slopes of the plateau and rocks of the Parauapebas Formation. Al-rich crusts may be formed from volcanic rocks, and can be associated with the occurrence of forest formations in the catchment of LTI1 and LTI3. Detritic and iron-ore duricrusts (namely 'canga') are dominant in the study site and extensively colonized by montane savanna vegetation. In Carajás, the occurrence of extensive duricrusts and low water retention allowed the widespread development of plant species adapted to nutrient and hydric stress and hindered the colonization of tree species (Skirycz et al., 2014). More information about plant-soil relationship in duricrusts can be found on Nunes et al. (2015).

Limnological investigation shows these lakes are weakly stratified, of polymictic type, and the trophic state varies from oligorophic to eutrophic. Waters in these lakes are mostly acidic $(\mathrm{pH}$ 3.6-6.2), with high total Fe (0.04-1.52 mg/L) and low EC (2-27 $\mu \mathrm{S} /$ $\mathrm{cm}$ ) (Sahoo et al., in press).

Area forests are composed mainly of Sacoglottis mattogrossensis Malme, Matayba arborescens (Aubl.) Radlk., Aparisthmium cordatum (A.Juss.) Baill., Myrcia splendens (Sw.) DC. and Vochysia haenkeana Mart. The canga vegetation is composed of Vellozia glochidea Pohl., Callisthene microphylla Warm, Mimosa acutistipula var. ferrea Barneby, Norantea goyasensis Cambess., Tibouchina aspera Aubl. and Ipomoea marabaensis D.Austin \& Secco. Nymphoides humboldtiana (Kunh) Kuntze, and a macrophyte ( $N$. humboldtiana) with submerged roots and floating leaves, can be observed near the margins of LTI1 (ITV, 2016).

The regional climate is typically tropical monsoon, with average annual temperature of around $26^{\circ} \mathrm{C}$ (Alvares et al., 2013). The total mean annual precipitation is $\sim 1550 \mathrm{~mm}$ during the rainy season (November to May) and $350 \mathrm{~mm}$ during the dry season (June to October) (Moraes et al., 2005). 

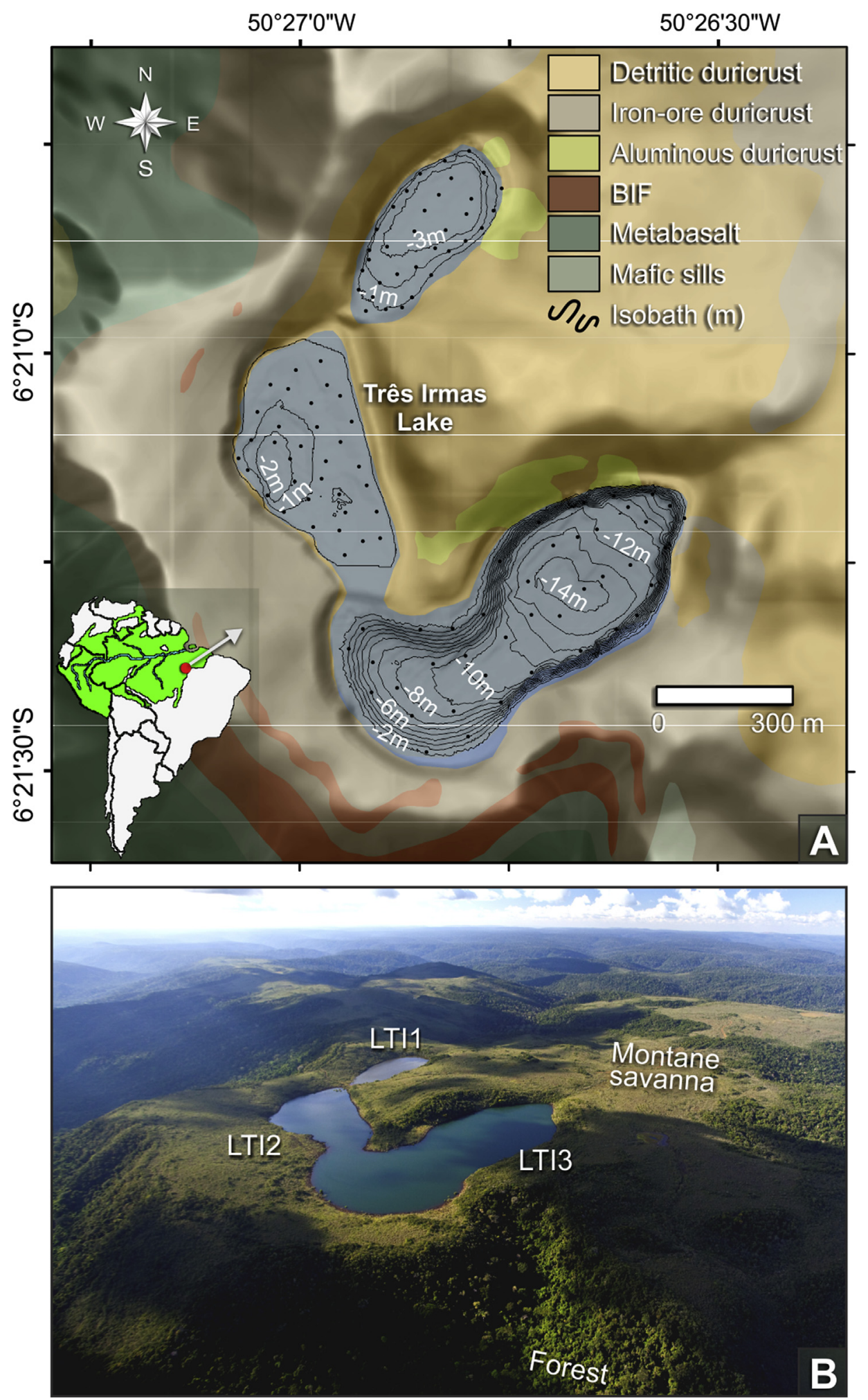

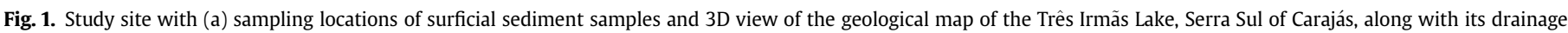

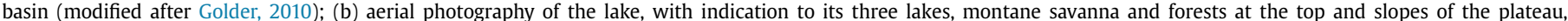
respectively. 


\section{Materials and methods}

\subsection{Sampling and analytical techniques}

A total of 80 surface sediment samples were collected from Três Irmãs Lake in March 2015 using a Van Veen Grab sampler, and geographical coordinates were obtained from a Trimble model AG132 differential GPS (DGPS) using differential correction from the OmniSTAR satellite ASAT L1 band. Sampling points were chosen based on bathymetry (Fig. 1A). After collection, sediment samples were placed in sealable plastic bags, labeled and brought to the laboratory for drying and grinding $<75 \mu$ for bulk geochemical analysis. Leaves of plants in the catchment basin were sampled for isotopic determination $\left(\delta^{13} \mathrm{C}\right.$ and $\left.\delta^{15} \mathrm{~N}\right)$ and elemental ratios $(\mathrm{C} / \mathrm{N})$ based on main vegetation types.

In laboratory, leaves (triturated and washed) and standards are weighed into tin cups, with weights dependent on the \% $\mathrm{C}$ and \% $\mathrm{N}$ of the sample material, but generally with a target weight of $0.3 \mathrm{mg}$. Additionally, we collected aluminous lateritic soil (Al-Soil) and AlCrusts around the lake catchment. Geochemical compositions of catchment lateritic crust, BIF and lateritic soil (L-Soil) used in this paper were determined by Sahoo et al. (2015).

Sediments were classified following Schnurrenberger et al. (2003) and facies described according to Walker (1992). Isotopic signatures $\left(\delta^{13} \mathrm{C}\right.$ and $\left.\delta^{15} \mathrm{~N}\right)$ of sediment and leaves were determined at the Center of Stable Isotopes, São Paulo State University (UNESP) and Center for Nuclear Energy in Agriculture (CENA), São Paulo University, using an elemental analyzer coupled to a continuous flow isotopic ratio mass spectrometer (CF-IRMS). Major and minor elements were determined by inductively coupled plasma optical emission spectrometry (ICP-OES) and trace and rare earth elements (REE) by inductively coupled plasma optical mass spectrometry (ICP-MS) after tetraborate fusion, by Acme Analytical Laboratory, Ltd., Canada. Total sulphur (TS) and total organic carbon (TOC) were measured using a LECO CS-300 combustion analyzer. Analytical accuracy and precision were checked using parallel analysis of standard reference materials (SRMs; GBM908-10, GRE 3, and OREAS 146) and duplicate measurements. The analytical accuracy was $\pm 10 \%$, indicating satisfactory recoveries and precision was better than $5 \%$.

\subsection{Statistical analysis}

Elemental associations (major, minor and REEs) in lake sediments were determined using Pearson $\mathrm{R}$ correlations. Critical values for the correlation coefficients at $\mathrm{p}<0.05$ were considered as highly significant. Principal component analysis (PCA) was used to determine relationships among variables and to simplify the data interpretation based on major controlling factors. This was carried utilizing the open source statistical software $\mathrm{R}$ version 3.1.2 ( $\mathrm{R}$ Development Core Team, 2014). All variables (major, trace and REEs concentrations) were log-transformed and standardized prior to PCA analysis in order to eliminate the influence of different measurement units and minimize the variances. In this study, Eigen values $>1$ were retained and varimax orthogonal rotation was used to transform initial matrices and to limit the number of variables loaded in each factor.

\section{Results}

\subsection{Sediment types and organic matter composition}

Três Irmâs Lake facies (Fig. 2a) include ferruginous gravels on the margins of LTI1 and LTI3, while the margins and center of the LTI2 are mainly composed of fragmental peat. This later facies was also observed in the marginal portions of LTI1 and LTI3 adjacent to ferruginous fragments. Organic mud was mainly restricted to central portions of the lakes, and highly dominated at LTI2 forming small ellipsoidal spots.

The isotopic and elemental values of leaves from each vegetation type are given in Table A1. Canga vegetation has $\delta^{13} \mathrm{C}$ values from $-26 \%$ (Norantea goyasensis Cambess.) to $-30.4 \%$ (Guapira opposita (Vell.) Reitz) and $\delta^{15} \mathrm{~N}$ from $3 \%$ (Guapira opposita (Vell.) Reitz) to $-1.4 \%$ (Mimosa acutistipula var. ferrea Barneby); C/N ranges from 57 (Norantea goyasensis Cambess.) to 14 (Guapira opposita (Vell.) Reitz). Forest values include $\delta^{13} \mathrm{C}$ from $-26.4 \%$ (Vochysia haenkeana Mart.) to -33.9\%o (Myrcia splendens (Sw.) DC.), $\delta^{15} \mathrm{~N}$ from 3.7\% (Matayba arborescens (Aubl.) Radlk.) to $-2.2 \%$ (Abuta grandifolia (Mart.) Sandwith), and C/N from 42 (Myrcia silvatica Barb. Rodr.) to 10 (Pleonotoma jasminifolia (Kunth) Miers).

Sediment samples of LTI1 for $\delta^{13} \mathrm{C}$ and $\delta^{15} \mathrm{~N}$ varied from -25.1 to $-29.3 \%$, and from 5 to $1 \%$ (Fig. $2 a$ ), respectively, which suggests the organic matter is derived from C3 vascular plants $\left(\delta^{13} \mathrm{C}\right.$ from -21 to $-32 \%$, Deines, 1980 ; Lamb et al., 2006; $\delta^{15} \mathrm{~N}-0 \%$, Thornton and McManus, 1994; Meyers, 1997; Sahoo et al., 2015), and/or from macrophytes and algae $\left(\delta^{13} \mathrm{C}\right.$ from -25 to $-30 \%$, Smith et al., 2012; Sahoo et al., 2016, 2016; $\delta^{15} \mathrm{~N}$ from 6 to 3\%o, Troxler and Richards, 2009; $\delta^{15} \mathrm{~N}-6 \%$ ). Additionally, sediment $\mathrm{C} / \mathrm{N}$ values from 12 to 22 have been attributed to a mixture of terrestrial plants and freshwater DOC (Tyson, 1995; Meyers, 1994). The $\delta^{13} \mathrm{C}$ and $\delta^{15} \mathrm{~N}$ values for LTI2 range from -27.2 to $-30.3 \%$, and $-0.2-2.5 \%$, respectively, and may be from $\mathrm{C} 3$ plants, while $\mathrm{C} / \mathrm{N}$ values from 11 to 33 are indicative of partial contribution from freshwater DOC. Sediments of LTI3 show $\delta^{13} \mathrm{C}$ and $\delta^{15} \mathrm{~N}$ values from -25.9 to $-27.9 \%$, and 6 to $4.2 \%$, respectively, which may be associated with mixed sources including terrestrial plants, macrophytes and algae. In addition, $\mathrm{C} / \mathrm{N}$ values from 12 to 26 indicate a mixture from terrestrial plants and freshwater DOC, similar to LTI1 (Fig. 2a).

The binary diagram for $\delta^{13} \mathrm{C}$ and $\mathrm{C} / \mathrm{N}$ (Fig. 3a) reinforces the predominant contribution of $\mathrm{C} 3$ plants and freshwater DOC, while the relation between $\delta^{15} \mathrm{~N}$ and $\delta^{13} \mathrm{C}$ points to higher influence from aquatic organic matter for LTI1 and LTI3 (Fig. 3b). The main isotopic signals observed for LTI2 are related to C3 vascular plants, and are very similar with those observed in swamps (filled lake) over ferruginous crusts in the study site (Sahoo et al., 2016). Scatterplots using isotopic and elemental ratios of plants from the main vegetation types (Fig. $3 c$ and d), and multivariate data analysis (Fig. 3e) may indicate that sedimentary organic matter has provided a higher contribution compared to forests, but the higher values of $\delta^{15} \mathrm{~N}$ also suggest dilution with organic matter.

\subsection{Characteristics of major elements}

Major oxide concentrations in Três Irmâs lake sediments are summarized in Table 1 along with the compositions of Upper Continental Crust (UCC) (Wedepohl, 1995), Post-Archean Australian Shales (PAAS) (Turekian and Wedepohl, 1961), and catchment materials such as lateritic crusts (LC), Banded iron formation (BIF) and lateritic soil (L-Soil) (Sahoo et al., 2015) and aluminous lateritic soil (Al-Soil) and Al-crust. Spatial distribution of major elements is presented in Fig. 2b. The mean total concentrations of major elements in the sediments decrease in the order $\mathrm{Fe}>\mathrm{Si}>\mathrm{Al}>\mathrm{Ti}>\mathrm{K}>$ ( $\mathrm{Ca}, \mathrm{Mg}, \mathrm{Na}$, and $\mathrm{K}$ ). As expected, $\mathrm{Fe}_{2} \mathrm{O}_{3}$ is extremely high in the sediments due to ferruginous laterites of the catchment. The highest concentrations of $\mathrm{Fe}_{2} \mathrm{O}_{3}$ occurred in LTI3 (28-63\%) mainly in the southwestern sector of the lake, followed by LTI1 (1.3-60.1\%), where the maximum enrichment occurred in the northwestern margin of lake. The $\mathrm{Fe}_{2} \mathrm{O}_{3}$ in LTI2 was ranged from 1.3 to $18.7 \%$. Although $\mathrm{Fe}_{2} \mathrm{O}_{3}$ is positively correlated with $\mathrm{Al}_{2} \mathrm{O}_{3}$ $(\mathrm{r}=0.69)$ (Table 2), $\mathrm{Fe}_{2} \mathrm{O}_{3}$ and $\mathrm{Al}_{2} \mathrm{O}_{3}$ are differently distributed. 

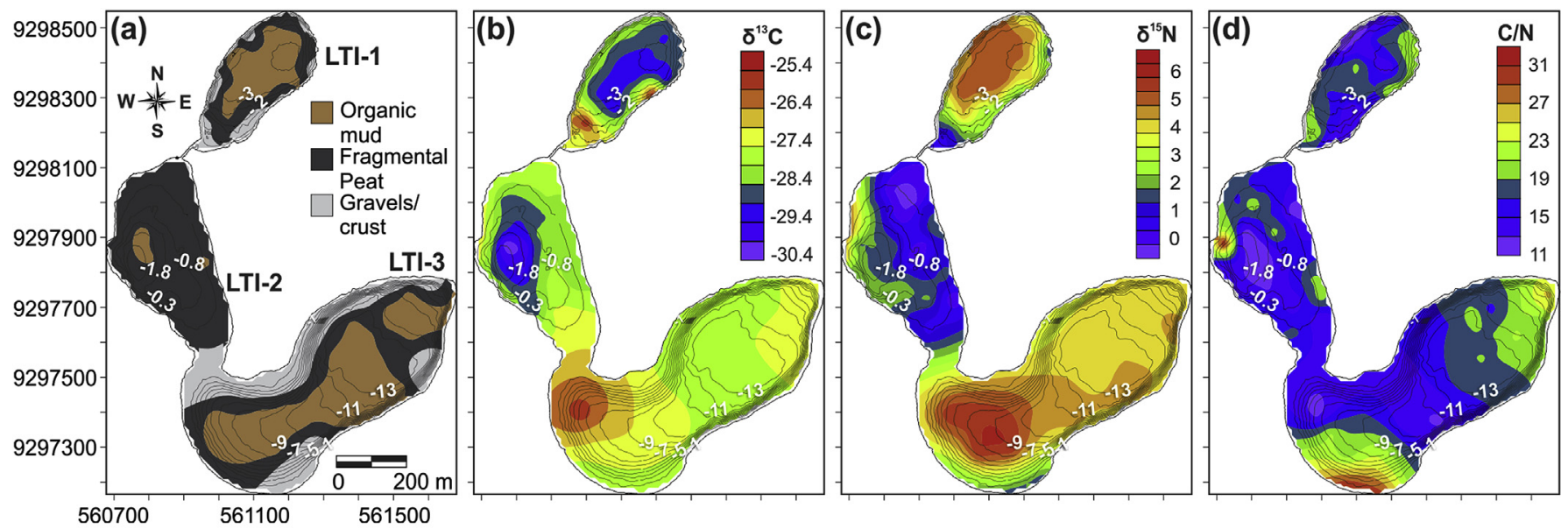

a
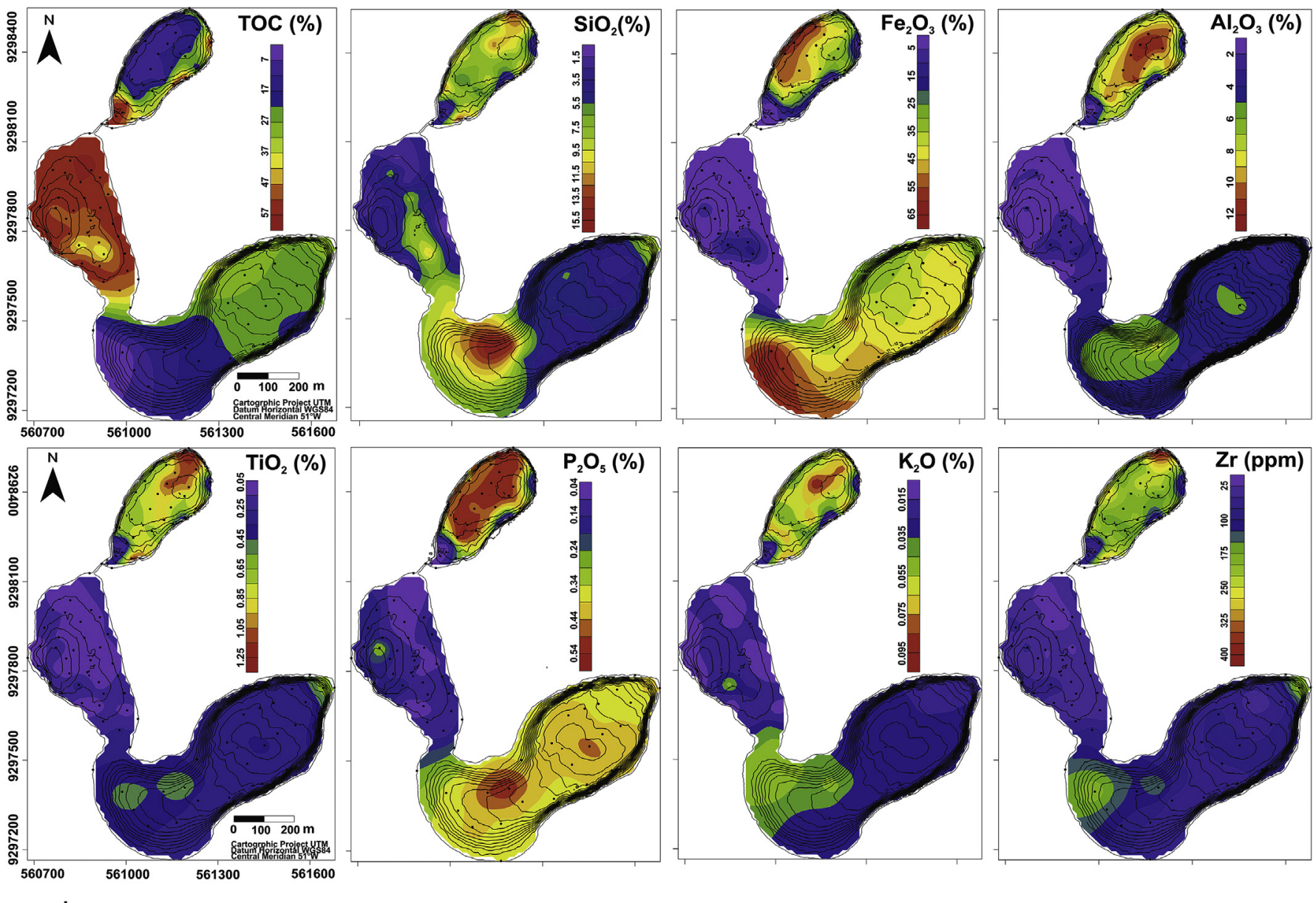

b

Fig. 2. (a) Facies description and spatial distribution of organic and (b) inorganic components in surface sediments $\left(\delta^{15} \mathrm{~N}\right.$ and $\delta^{13} \mathrm{C}$ are expressed as delta per mil, \%o).

$\mathrm{Al}_{2} \mathrm{O}_{3}$ content ranged from 1.72 to $12.7 \%, 0.8-4.13 \%$ and $3.45-7.6 \%$ in LTI1, LTI2, and LTI3, respectively. The highest occurrence of $\mathrm{Al}_{2} \mathrm{O}_{3}$ and $\mathrm{K}_{2} \mathrm{O}$ in LTI1 was noted at the center of the lake towards the northern part, which corresponds to the catchment with Al-rich lateritic soils, while at $\mathrm{LTI} 3 \mathrm{Al}_{2} \mathrm{O}_{3}$ is mainly concentrated on the southwestern margins. Similar distribution and the strong correlation between $\mathrm{Al}_{2} \mathrm{O}_{3}$ and $\mathrm{K}_{2} \mathrm{O}(\mathrm{r}=0.92)$ suggest that the two elements are related in their mode of deposition and have a common origin. The concentration of $\mathrm{SiO}_{2}$ varies from 1.26 to $13.7 \%$, $1.3-11.23 \%$ and $2.99-15.65 \%$ in LTI1, LTI2, and LTI3, respectively. The spatial distribution of $\mathrm{SiO}_{2}$ shows a marked enrichment at the northern border of LTI1 and southwestern portion of LTI3. The distribution of $\mathrm{TiO}_{2}$ is similar to $\mathrm{SiO}_{2}$, and varies from 0.15 to $1.26 \%$, $0.06-0.333 \%$ and $0.25-0.63 \%$ in LTI1, LTI2, and LTI3, respectively. 
(a)

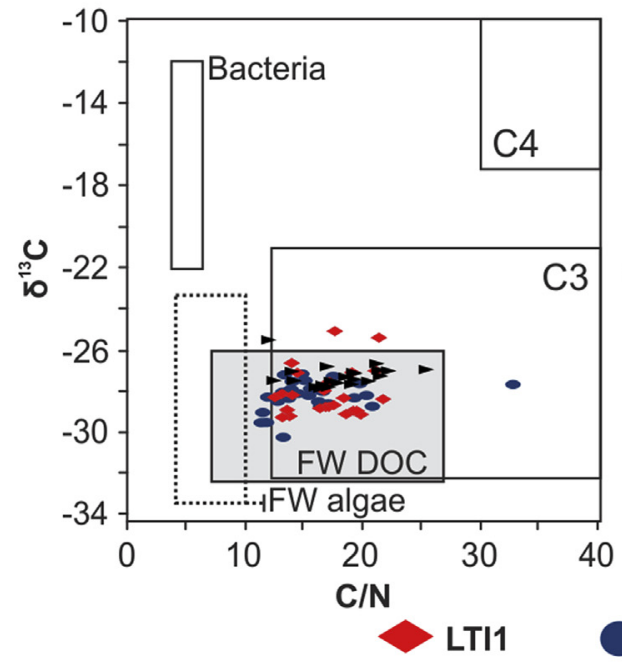

(c)

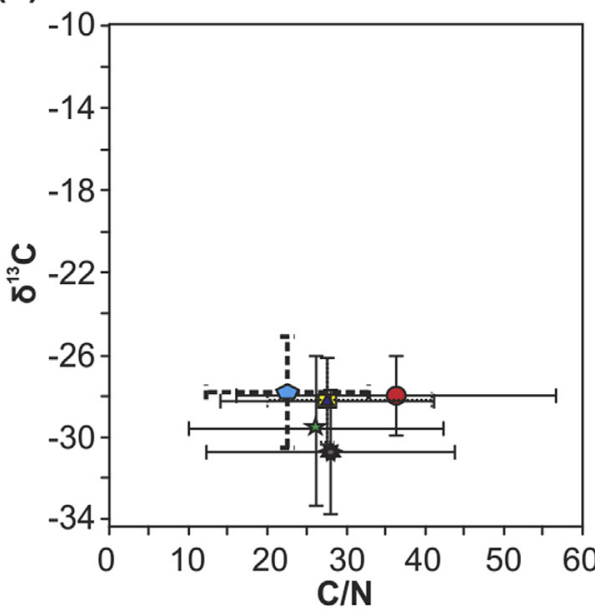

MS1

$\square \mathrm{MS} 2$

$\triangle \mathrm{MS} 3$

茓FF LTI3

FF LTI1

Sediment (b)

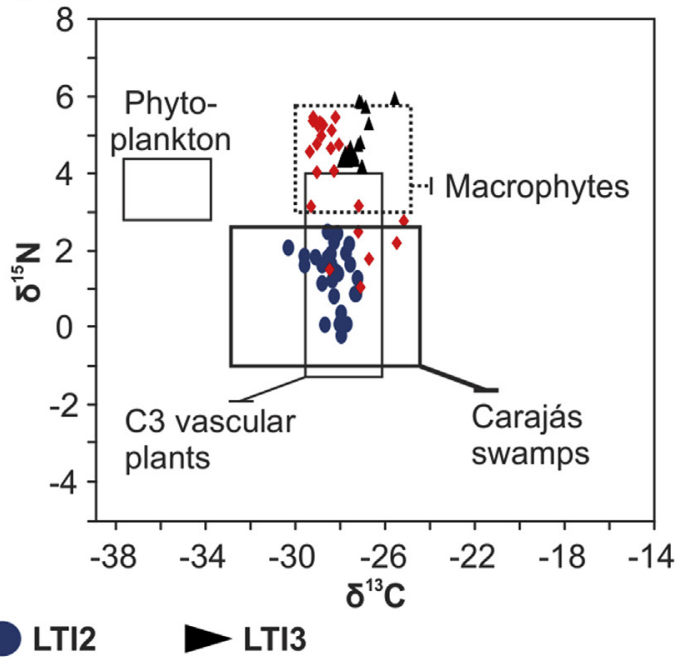

(d)

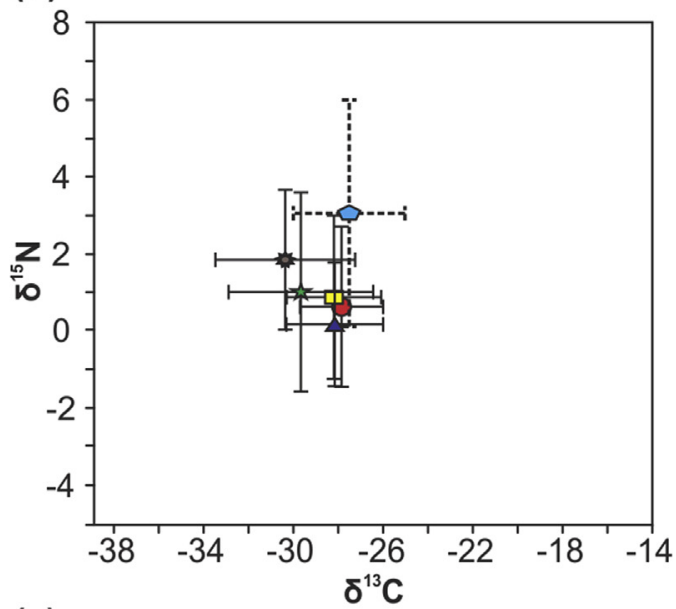

(e)

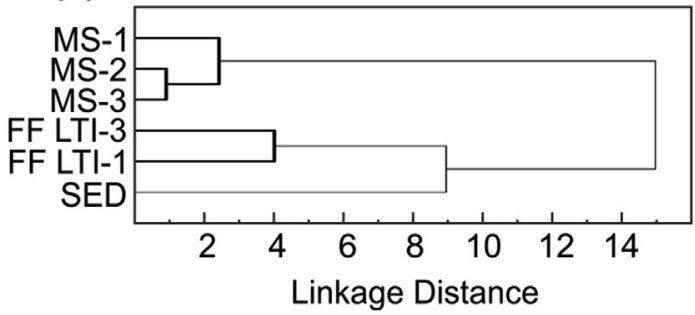

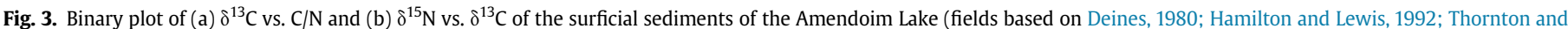

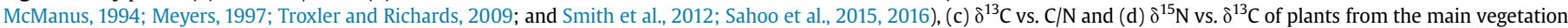

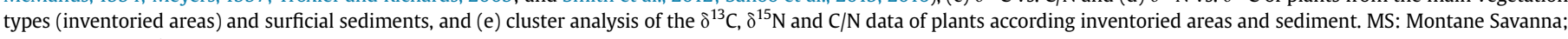
FF: Forest Formation.

Positive $\mathrm{Al}_{2} \mathrm{O}_{3}-\mathrm{TiO}_{2}$ correlation $(\mathrm{r}=0.93)$ and weak $\mathrm{TiO}_{2}-\mathrm{Fe}_{2} \mathrm{O}_{3}$ correlation $(\mathrm{r}=0.5)$ indicates that $\mathrm{TiO}_{2}$ distribution is mainly controlled by Al-clays. Strong positive correlation between $\mathrm{SiO}_{2}-$ $\mathrm{Al}_{2} \mathrm{O}_{3}(\mathrm{r}=0.67)$ and negative correlation between $\mathrm{SiO}_{2}$-TOC $(-0.59)$ indicates that $\mathrm{Si}$ is mostly inorganically bound. Positive correlation between $\mathrm{SiO}_{2}-\mathrm{TiO}_{2}(\mathrm{r}=0.69)$ and $\mathrm{SiO}_{2}-\mathrm{Zr}(\mathrm{r}=0.72)$ indicates that heavy minerals control their distribution. The concentrations of the remaining major oxides $\left(\mathrm{CaO}, \mathrm{MgO}\right.$ and $\left.\mathrm{Na}_{2} \mathrm{O}\right)$ were generally $<0.5 \mathrm{wt} \%$. Poor correlation coefficients for these oxides with $\mathrm{Al}_{2} \mathrm{O}_{3}$ indicates they were not controlled by Al-clays. The concentration of TOC varies between the lakes and shows a strong spatial variation; the highest concentration was found throughout LIT2 (36.7-59\%), followed by LTI1 (6.8-57.2\%) and LTI3 (3.6-29.5\%). By comparison with the average Upper Continental Crust (UCC) (Wedepohl, 1995) (Fig. 4), the sediments (except TLI2) are mainly enriched in $\mathrm{Fe}_{2} \mathrm{O}_{3}$, and $\mathrm{P}_{2} \mathrm{O}_{5}$, but are depleted in all other major oxides. Similar enrichment patterns were observed for catchment materials such as laterite crust (LC) and lateritic soil (LSoil), while all elements are depleted relative to BIF. Both Al-Crust and aluminous lateritic soil (Al-Soil) are enriched in $\mathrm{Fe}_{2} \mathrm{O}_{3}, \mathrm{P}_{2} \mathrm{O}_{5}$, and $\mathrm{TiO}_{2}$, while $\mathrm{Al}_{2} \mathrm{O}_{3}$ is only enriched in $\mathrm{Al}-\mathrm{Crust}$. $\mathrm{TiO}_{2}$ enrichment was also observed in TLI1 (Fig. 4). 
Table 1

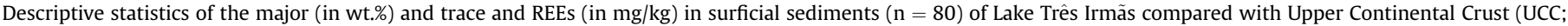

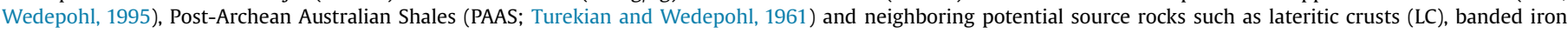
formation (BIF), Al-Crust, lateritic soil (L-Soil), and aluminous lateritic soil (Al-Soil); LC, BIF and L-Soil geochemical data were taken from Sahoo et al. (2015).

\begin{tabular}{|c|c|c|c|c|c|c|c|c|c|c|c|c|c|c|c|c|}
\hline & \multicolumn{3}{|l|}{ LTI1 } & \multicolumn{3}{|l|}{ LTI2 } & \multicolumn{3}{|l|}{ LTI3 } & \multirow{2}{*}{$\frac{\text { Al-Soil }}{\text { Avg }}$} & \multirow[t]{2}{*}{ L-Soil } & \multirow[t]{2}{*}{ LC } & \multirow[t]{2}{*}{ Al-Crust } & \multirow[t]{2}{*}{ BIF } & \multirow[t]{2}{*}{ PAAS } & \multirow[t]{2}{*}{ UCC } \\
\hline & Min & Max & Avg & Min & Max & Avg & Min & Max & Avg & & & & & & & \\
\hline $\mathrm{SiO}_{2}$ & 1.26 & 13.74 & 7.84 & 1.30 & 11.2 & 4.41 & 2.99 & 15.65 & 6.37 & 7.81 & 3.58 & 0.695 & 0.9 & 46.61 & 62.8 & 64.92 \\
\hline $\mathrm{TiO}_{2}$ & 0.15 & 1.26 & 0.76 & 0.06 & 0.33 & 0.15 & 0.25 & 0.63 & 0.36 & 2.82 & 0.43 & 0.275 & 1.07 & 0.01 & 1 & 0.52 \\
\hline $\mathrm{Al}_{2} \mathrm{O}_{3}$ & 1.72 & 12.73 & 7.78 & 0.89 & 4.13 & 2.04 & 3.45 & 7.65 & 4.73 & 13.59 & 1.95 & 1.825 & 37.68 & 0.05 & 18.9 & 14.63 \\
\hline $\mathrm{Fe}_{2} \mathrm{O}_{3}$ & 1.32 & 60.18 & 31.97 & 1.37 & 18.7 & 4.02 & 28.08 & 63.90 & 43.24 & 53.36 & 83.41 & 92.27 & 37.52 & 50.71 & 6.5 & 4.42 \\
\hline $\mathrm{MnO}$ & $<0.01$ & 0.01 & 0.01 & $<0.01$ & 0.01 & 0.01 & $<0.01$ & 0.01 & 0.01 & 0.03 & 0.02 & 0.025 & $<0.01$ & 0.02 & 0.11 & 0.07 \\
\hline $\mathrm{MgO}$ & $<0.01$ & 0.05 & 0.02 & $<0.01$ & 0.03 & 0.02 & $<0.01$ & 0.02 & 0.01 & 0.02 & $<0.01$ & $<0.01$ & 0.02 & 0.07 & 2.2 & 2.24 \\
\hline $\mathrm{CaO}$ & $<0.01$ & 0.03 & 0.02 & $<0.01$ & 0.08 & 0.03 & $<0.01$ & 0.06 & 0.02 & 0.02 & $<0.01$ & $<0.01$ & 0.06 & $<0.01$ & 1.3 & 4.12 \\
\hline $\mathrm{Na}_{2} \mathrm{O}$ & $<0.01$ & 0.01 & 0.01 & $<0.01$ & 0.01 & 0.01 & $<0.01$ & 0.01 & 0.01 & $<0.01$ & $<0.01$ & $<0.01$ & $<0.01$ & $<0.01$ & 1.2 & 3.46 \\
\hline $\mathrm{K}_{2} \mathrm{O}$ & $<0.01$ & 0.1 & 0.06 & $<0.01$ & 0.05 & 0.02 & 0.02 & 0.05 & 0.03 & 0.02 & $<0.01$ & $<0.01$ & $<0.01$ & $<0.01$ & 3.7 & 3.45 \\
\hline $\mathrm{P}_{2} \mathrm{O}_{5}$ & 0.06 & 0.56 & 0.39 & 0.04 & 0.33 & 0.12 & 0.34 & 0.55 & 0.40 & 0.67 & 0.77 & 0.62 & 0.68 & $<0.01$ & 0.16 & 0.15 \\
\hline TOC & 6.84 & 57.29 & 24.42 & 36.74 & 59.1 & 52.17 & 3.66 & 29.57 & 21.34 & 7.57 & & & & & & \\
\hline TOS & 0.02 & 0.31 & 0.12 & 0.09 & 0.33 & 0.16 & 0.02 & 0.15 & 0.06 & 0.06 & & & & & & \\
\hline \multirow[t]{3}{*}{ LOI } & 25.4 & 95.2 & 51.07 & 66.60 & 95.20 & 89.15 & 19.50 & 57.60 & 44.75 & 21.5 & & & & & & \\
\hline & \multicolumn{3}{|l|}{ LTI1 } & LTI2 & & & LTI3 & & & Al-Soil & L-Soil & LC & Al-Crust & $\mathrm{BIF}$ & PAAS & UCC \\
\hline & Min & Max & Avg & Min & Max & Avg & Min & Max & Avg & Avg & & & & & & \\
\hline $\mathrm{Ba}$ & 8 & 91 & 26.33 & 6.00 & 30.0 & 13.25 & 8.00 & 23.00 & 14.52 & 11.00 & 16 & 20.5 & 4.9 & 7 & 580 & 668 \\
\hline $\mathrm{Sr}$ & 2.1 & 11.9 & 7.36 & 2.30 & 6.50 & 3.46 & 2.80 & 7.40 & 4.38 & 6.17 & 5.5 & 9.05 & 0.6 & 0.7 & 300 & 316 \\
\hline $\mathrm{Rb}$ & 0.3 & 3.9 & 2.11 & 0.20 & 2.10 & 0.64 & 0.70 & 2.00 & 1.10 & 0.37 & 0.5 & 0.01 & 0.1 & 0.8 & 140 & 110 \\
\hline $\mathrm{Pb}$ & 5.5 & 28.8 & 17.24 & 2.80 & 12.9 & 4.91 & 7.00 & 12.50 & 8.94 & 12.27 & 13.9 & 5.35 & 1.7 & 2.1 & 20 & 17 \\
\hline Sc & 3 & 13 & 8.88 & 1.00 & 5.00 & 2.25 & 4.00 & 9.00 & 5.74 & 24.00 & 3 & 6 & 26.1 & 1 & 16 & 7 \\
\hline Th & 2.2 & 14.8 & 9.68 & 1.10 & 5.30 & 2.70 & 4.20 & 8.00 & 5.36 & 14.17 & 4.7 & 6.9 & 9.37 & 0.2 & 12 & 10 \\
\hline $\mathrm{U}$ & 0.4 & 3.2 & 1.51 & 0.10 & 0.70 & 0.38 & 0.50 & 1.30 & 0.88 & 2.67 & 1 & 0.6 & 1.28 & 0.1 & 3.7 & 2.5 \\
\hline $\mathrm{Zr}$ & 41 & 443 & 208.54 & 15.00 & 95.0 & 40.03 & 58.00 & 220.0 & 96.65 & 468.60 & 140.3 & 71.05 & 157 & 1 & 160 & 237 \\
\hline $\mathrm{Hf}$ & 0.9 & 11.8 & 5.06 & 0.40 & 2.40 & 1.03 & 1.00 & 5.20 & 2.39 & 12.33 & 3.8 & 1.95 & 4.3 & 0.1 & 2.8 & 5.8 \\
\hline $\mathrm{Nb}$ & 2.5 & 19.6 & 12.13 & 1.10 & 6.60 & 2.50 & 1.80 & 9.40 & 5.53 & 26.93 & 8.4 & 5 & 8.1 & 0.1 & 19 & 26 \\
\hline Y & 2.1 & 15.2 & 9.61 & 1.10 & 6.30 & 2.72 & 3.80 & 8.10 & 5.71 & 10.93 & 5.4 & 4.9 & 4.6 & 2.6 & 27 & 20.7 \\
\hline $\mathrm{Cu}$ & 19.7 & 84.3 & 32.69 & 8.50 & 24.3 & 14.44 & 16.70 & 37.70 & 27.82 & 60.23 & 18.8 & 12.85 & 78.9 & 11.6 & 45 & 14 \\
\hline $\mathrm{Zn}$ & 2 & 55 & 29.21 & 1.00 & 31.0 & 7.28 & 31.00 & 70.00 & 48.00 & 25.33 & 29 & 19.5 & 97 & 16 & 95 & 52 \\
\hline As & 0.5 & 4.1 & 1.66 & 0.50 & 1.70 & 0.56 & 0.50 & 3.10 & 1.14 & 3.70 & 6.6 & 7.55 & 3.4 & 0.6 & 13 & 2 \\
\hline $\mathrm{Se}$ & 3 & 7.5 & 5.12 & 1.40 & 7.30 & 4.67 & 3.00 & 7.10 & 5.38 & 0.67 & 1.1 & 0.5 & 0.2 & 0.5 & 0.6 & 0.1 \\
\hline Mo & 0.5 & 4.3 & 2.65 & 0.30 & 1.70 & 0.72 & 1.20 & 2.90 & 1.83 & 1.93 & 1.3 & 1.9 & 0.68 & 0.5 & 2.6 & 1.4 \\
\hline $\mathrm{Hg}$ & 0.08 & 0.31 & 0.18 & 0.15 & 0.35 & 0.24 & 0.10 & 0.28 & 0.22 & 0.12 & 0.17 & 0.045 & 0.12 & 0.01 & 0.4 & 0.06 \\
\hline V & 23 & 160 & 108.04 & 18.00 & 94.0 & 30.25 & 26.00 & 107.0 & 61.39 & 374.00 & 42 & 63 & 167 & 19 & 130 & 53 \\
\hline $\mathrm{Cr}$ & 20 & 140 & 91.25 & 20.00 & 50.0 & 25.31 & 50.00 & 90.00 & 63.91 & 193.33 & 80 & 85.5 & 250 & 34 & 90 & 35 \\
\hline Ga & 2.7 & 23 & 14.54 & 1.20 & 6.80 & 3.57 & 4.10 & 11.70 & 7.44 & 36.47 & 8.1 & 8.65 & 24.2 & 0.5 & 19 & 14 \\
\hline Co & 0.4 & 2 & 0.98 & 0.20 & 1.00 & 0.45 & 0.60 & 1.70 & 1.03 & 3.73 & 1.2 & 0.7 & 1.3 & 0.4 & 19 & 12 \\
\hline $\mathrm{Ni}$ & 2.7 & 9.7 & 5.48 & 1.30 & 6.00 & 3.21 & 3.20 & 6.90 & 5.34 & 10.80 & 1.5 & 20 & 63.6 & 20 & 68 & 18.6 \\
\hline & LTI1 & & & LTI2 & & & LTI3 & & & Al-Soil & L-Soil & LC & Al-Crust & $\mathrm{BIF}$ & PAAS & UCC \\
\hline & Min & Max & Avg & Min & Max & Avg & Min & Max & Avg & Avg & & & & & & \\
\hline $\mathrm{La}$ & 5.4 & 28 & 16.79 & 3.50 & 10.5 & 5.98 & 7.80 & 14.50 & 10.71 & 22.33 & 6.2 & 7.55 & 12.4 & $\overline{1.9}$ & 38 & 30 \\
\hline $\mathrm{Ce}$ & 9.6 & 54.6 & 31.88 & 5.80 & 22.4 & 11.18 & 16.40 & 28.90 & 20.85 & 32.77 & 13.4 & 26.95 & 20.6 & 1.5 & 80 & 64 \\
\hline $\operatorname{Pr}$ & 0.91 & 5.3 & 3.28 & 0.56 & 2.25 & 1.14 & 1.65 & 3.09 & 2.17 & 4.67 & 1.11 & 1.58 & 2.22 & 0.16 & 8.9 & 7.1 \\
\hline $\mathrm{Nd}$ & 3.3 & 19.1 & 11.70 & 2.00 & 8.10 & 4.14 & 5.60 & 11.10 & 7.57 & 16.63 & 4.1 & 5.55 & 7.8 & 0.8 & 32 & 26 \\
\hline $\mathrm{Sm}$ & 0.49 & 3.49 & 2.05 & 0.30 & 1.65 & 0.73 & 0.88 & 1.87 & 1.34 & 2.73 & 0.63 & 0.875 & 1.66 & 0.09 & 5.6 & 4.5 \\
\hline $\mathrm{Eu}$ & 0.11 & 0.77 & 0.45 & 0.07 & 0.30 & 0.15 & 0.19 & 0.37 & 0.30 & 0.66 & 0.2 & 0.215 & 0.43 & 0.1 & 1.1 & 0.88 \\
\hline Gd & 0.42 & 2.87 & 1.80 & 0.29 & 1.21 & 0.61 & 0.91 & 1.68 & 1.18 & 1.96 & 0.65 & 0.865 & 1.24 & 0.06 & 4.66 & 3.5 \\
\hline $\mathrm{Tb}$ & 0.06 & 0.45 & 0.28 & 0.04 & 0.17 & 0.09 & 0.12 & 0.23 & 0.18 & 0.31 & 0.11 & 0.16 & 0.22 & 0.01 & 0.77 & 0.64 \\
\hline Dy & 0.39 & 2.86 & 1.72 & 0.15 & 1.08 & 0.51 & 0.66 & 1.52 & 1.01 & 1.94 & 0.68 & 0.64 & 1.21 & 0.13 & 4.4 & 3.5 \\
\hline Ho & 0.07 & 0.53 & 0.35 & 0.04 & 0.21 & 0.10 & 0.14 & 0.29 & 0.20 & 0.42 & 0.15 & 0.135 & 0.29 & 0.02 & 1 & 0.8 \\
\hline $\mathrm{Er}$ & 0.19 & 1.72 & 1.08 & 0.12 & 0.62 & 0.29 & 0.43 & 0.88 & 0.59 & 1.35 & 0.56 & 0.365 & 1.08 & 0.06 & 2.9 & 2.3 \\
\hline $\mathrm{Tm}$ & 0.03 & 0.27 & 0.17 & 0.01 & 0.10 & 0.04 & 0.05 & 0.16 & 0.10 & 0.24 & 0.09 & 0.075 & 0.15 & 0.03 & 0.4 & 0.33 \\
\hline $\mathrm{Yb}$ & 0.25 & 1.73 & 1.13 & 0.09 & 0.72 & 0.29 & 0.40 & 0.93 & 0.59 & 1.75 & 0.62 & 0.255 & 1.63 & 0.07 & 2.8 & 2.2 \\
\hline $\mathrm{Lu}$ & 0.03 & 0.28 & 0.18 & 0.02 & 0.09 & 0.04 & 0.05 & 0.14 & 0.10 & 0.30 & 0.11 & 0.01 & 0.22 & 0.02 & 0.43 & 0.32 \\
\hline LREE & 20.2 & 110 & 65.7 & 12.1 & 39.8 & 23.6 & 33.6 & 59.1 & 42.6 & 79.1 & 25.4 & 42.5 & 44.6 & 4.45 & 165 & 132 \\
\hline HREE & 1.48 & 10.45 & 6.69 & 0.77 & 4.21 & 2.03 & 2.89 & 5.83 & 3.95 & 8.27 & 2.97 & 2.50 & 6.04 & 0.4 & 17.4 & 13.6 \\
\hline$\Sigma$ REE & 21.84 & 121.71 & 72.85 & 13.00 & 49.4 & 25.28 & 35.71 & 65.36 & 46.90 & 88.06 & 28.61 & 45.23 & 51.15 & 4.95 & 182.9 & 146.0 \\
\hline
\end{tabular}

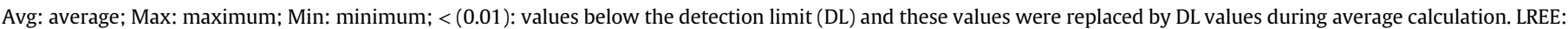
Light Rare Earth Elements; HREE: Heavy Rare Earth Elements.

The geochemical classification of sediments was made using the Herron plot (Herron, 1988) of $\log \left(\mathrm{Fe}_{2} \mathrm{O}_{3} / \mathrm{K}_{2} \mathrm{O}\right)$ vs. $\log \left(\mathrm{SiO}_{2} / \mathrm{Al}_{2} \mathrm{O}_{3}\right)$ (Fig. 5). The $\log$ ratio of $\mathrm{SiO}_{2} / \mathrm{Al}_{2} \mathrm{O}_{3}$ indicates the maturity of sediments, and it differentiates between quartz-rich high ratio sandstone and clay -rich low ratio shale. This analysis shows that most of the sediment samples occur in the Fe-shale field. The mineralogy of the sediments further evaluated by the $\mathrm{K}_{2} \mathrm{O} / \mathrm{Al}_{2} \mathrm{O}_{3}$ ratio, this ratio ranges from 0.4 to 1 for alkali feldspar, approximately 0.3 for illite, and $<0.3$ for clay minerals (Cox et al., 1995). In our study, the $\mathrm{K}_{2} \mathrm{O} / \mathrm{Al}_{2} \mathrm{O}_{3}$ ratio was $<0.3$ in all samples, suggesting the dominance of clay minerals. On the $\mathrm{Fe}_{2} \mathrm{O}_{3}-\mathrm{SiO}_{2}-\mathrm{Al}_{2} \mathrm{O}_{3}$ triangular plot (Fig. 6a) (Schellmann, 1981; Schellmann, 1986), which is used to classify laterites, catchment 
Table 2

Pearson correlation coefficient between major and trace elements in Três Irmãs lake sediments $(\mathrm{n}=80)$.

\begin{tabular}{|c|c|c|c|c|c|c|c|c|c|c|c|c|c|c|}
\hline & CIA & IOL & MIA & $\mathrm{Si}$ & $\mathrm{Ti}$ & $\mathrm{Al}$ & $\mathrm{Fe}$ & $\mathrm{Mg}$ & $\mathrm{Ca}$ & K & $\mathrm{P}$ & TOC & $\mathrm{Zr}$ & $\mathrm{Hf}$ \\
\hline $\mathrm{CIA}$ & 1.00 & & & & & & & & & & & & & \\
\hline IOL & 0.57 & 1.00 & & & & & & & & & & & & \\
\hline MIA & 0.91 & 0.77 & 1.00 & & & & & & & & & & & \\
\hline $\mathrm{Si}$ & 0.53 & 0.08 & 0.52 & 1.00 & & & & & & & & & & \\
\hline $\mathrm{Ti}$ & 0.59 & 0.42 & 0.56 & 0.69 & 1.00 & & & & & & & & & \\
\hline $\mathrm{Al}$ & 0.69 & 0.55 & 0.68 & 0.67 & 0.93 & 1.00 & & & & & & & & \\
\hline $\mathrm{Fe}$ & 0.70 & 0.85 & 0.85 & 0.44 & 0.56 & 0.69 & 1.00 & & & & & & & \\
\hline $\mathrm{Mg}$ & -0.23 & -0.43 & -0.39 & 0.27 & 0.35 & 0.18 & -0.41 & 1.00 & & & & & & \\
\hline $\mathrm{Ca}$ & -0.73 & -0.29 & -0.60 & -0.28 & -0.22 & -0.33 & -0.41 & 0.31 & 1.00 & & & & & \\
\hline K & 0.58 & 0.38 & 0.57 & 0.76 & 0.92 & 0.92 & 0.57 & 0.35 & -0.32 & 1.00 & & & & \\
\hline $\mathrm{P}$ & 0.69 & 0.78 & 0.81 & 0.53 & 0.75 & 0.84 & 0.87 & -0.13 & -0.25 & 0.70 & 1.00 & & & \\
\hline TOC & -0.73 & -0.78 & -0.85 & -0.59 & -0.70 & -0.82 & -0.97 & 0.25 & 0.40 & -0.72 & -0.92 & 1.00 & & \\
\hline $\mathrm{Zr}$ & 0.54 & 0.36 & 0.52 & 0.72 & 0.97 & 0.84 & 0.53 & 0.34 & -0.21 & 0.87 & 0.70 & -0.67 & 1.00 & \\
\hline $\mathrm{Hf}$ & 0.53 & 0.35 & 0.51 & 0.72 & 0.96 & 0.82 & 0.52 & 0.35 & -0.21 & 0.87 & 0.69 & -0.66 & 0.99 & 1.00 \\
\hline $\mathrm{Ba}$ & 0.35 & 0.15 & 0.28 & 0.58 & 0.70 & 0.68 & 0.23 & 0.52 & -0.12 & 0.76 & 0.40 & -0.38 & 0.65 & 0.64 \\
\hline $\mathrm{Sr}$ & 0.37 & 0.29 & 0.36 & 0.65 & 0.92 & 0.86 & 0.38 & 0.52 & 0.01 & 0.89 & 0.64 & -0.55 & 0.86 & 0.86 \\
\hline $\mathrm{Rb}$ & 0.58 & 0.39 & 0.57 & 0.70 & 0.90 & 0.94 & 0.54 & 0.34 & -0.31 & 0.97 & 0.71 & -0.69 & 0.83 & 0.82 \\
\hline $\mathrm{Pb}$ & 0.59 & 0.44 & 0.55 & 0.59 & 0.95 & 0.91 & 0.54 & 0.32 & -0.22 & 0.86 & 0.75 & -0.67 & 0.88 & 0.87 \\
\hline Sc & 0.70 & 0.56 & 0.68 & 0.64 & 0.94 & 0.97 & 0.66 & 0.22 & -0.30 & 0.90 & 0.84 & -0.78 & 0.85 & 0.84 \\
\hline Th & 0.64 & 0.47 & 0.61 & 0.67 & 0.97 & 0.96 & 0.60 & 0.31 & -0.27 & 0.93 & 0.79 & -0.74 & 0.90 & 0.90 \\
\hline U & 0.61 & 0.43 & 0.58 & 0.67 & 0.96 & 0.89 & 0.55 & 0.34 & -0.20 & 0.87 & 0.75 & -0.69 & 0.92 & 0.92 \\
\hline $\mathrm{Nb}$ & 0.58 & 0.40 & 0.55 & 0.70 & 0.99 & 0.92 & 0.54 & 0.37 & -0.23 & 0.93 & 0.73 & -0.69 & 0.96 & 0.95 \\
\hline $\mathrm{Y}$ & 0.66 & 0.48 & 0.64 & 0.70 & 0.97 & 0.95 & 0.61 & 0.30 & -0.28 & 0.93 & 0.80 & -0.75 & 0.92 & 0.92 \\
\hline $\mathrm{Zn}$ & 0.61 & 0.79 & 0.81 & 0.47 & 0.48 & 0.59 & 0.91 & -0.37 & -0.31 & 0.51 & 0.86 & -0.89 & 0.48 & 0.47 \\
\hline As & 0.49 & 0.52 & 0.53 & 0.36 & 0.50 & 0.59 & 0.73 & -0.28 & -0.43 & 0.50 & 0.56 & -0.74 & 0.53 & 0.50 \\
\hline Se & 0.23 & 0.04 & 0.12 & -0.03 & 0.06 & 0.11 & 0.03 & -0.04 & -0.14 & 0.03 & 0.11 & -0.02 & -0.03 & -0.01 \\
\hline Mo & 0.67 & 0.63 & 0.72 & 0.61 & 0.82 & 0.94 & 0.80 & -0.05 & -0.41 & 0.84 & 0.85 & -0.89 & 0.74 & 0.73 \\
\hline V & 0.67 & 0.55 & 0.64 & 0.60 & 0.91 & 0.97 & 0.65 & 0.18 & -0.32 & 0.87 & 0.81 & -0.77 & 0.84 & 0.82 \\
\hline $\mathrm{Cr}$ & 0.70 & 0.60 & 0.73 & 0.69 & 0.92 & 0.98 & 0.76 & 0.12 & -0.35 & 0.91 & 0.87 & -0.87 & 0.85 & 0.84 \\
\hline $\mathrm{Ga}$ & 0.62 & 0.45 & 0.59 & 0.68 & 0.98 & 0.96 & 0.57 & 0.33 & -0.25 & 0.93 & 0.78 & -0.72 & 0.91 & 0.90 \\
\hline Co & 0.59 & 0.57 & 0.68 & 0.50 & 0.59 & 0.64 & 0.65 & -0.08 & -0.32 & 0.63 & 0.69 & -0.70 & 0.56 & 0.57 \\
\hline $\mathrm{Ni}$ & 0.71 & 0.54 & 0.74 & 0.58 & 0.66 & 0.76 & 0.61 & 0.03 & -0.42 & 0.74 & 0.73 & -0.69 & 0.56 & 0.57 \\
\hline LREE & 0.66 & 0.49 & 0.64 & 0.67 & 0.95 & 0.95 & 0.59 & 0.32 & -0.25 & 0.92 & 0.79 & -0.73 & 0.87 & 0.87 \\
\hline HREE & 0.65 & 0.47 & 0.63 & 0.71 & 0.98 & 0.96 & 0.61 & 0.32 & -0.28 & 0.94 & 0.79 & -0.75 & 0.93 & 0.92 \\
\hline $\mathrm{Ce} / \mathrm{Ce}^{*}$ & 0.08 & 0.12 & 0.18 & 0.20 & 0.11 & 0.14 & 0.19 & 0.07 & 0.09 & 0.18 & 0.20 & -0.20 & 0.10 & 0.09 \\
\hline $\mathrm{Eu} / \mathrm{Eu}^{*}$ & 0.05 & 0.07 & -0.70 & 0.08 & 0.07 & 0.09 & -0.09 & 0.07 & 0.25 & 0.06 & -0.05 & 0.07 & -0.08 & -0.05 \\
\hline
\end{tabular}

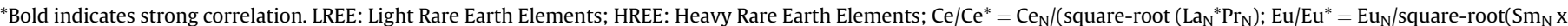
$\mathrm{Gd}_{\mathrm{N}, \mathrm{N}}$ : normalized to chondrite values (Schmitt et al., 1963).

materials are classified as ferruginous laterite, while sediments are classified as products of laterite. The $\mathrm{Fe}_{2} \mathrm{O}_{3}-\mathrm{SiO}_{2}-\mathrm{Al}_{2} \mathrm{O}_{3}$ plot (Fig. 6a, modified from Schellmann, 1986; Babechuk et al., 2014) can quantify the degree of laterization and shows that the catchment laterites are strongly laterized, while weathered sediments are range between strong and weak lateriazation. The degree of weathering was calculated with the Chemical Index of Alteration ( $\mathrm{CIA}=\mathrm{Al}_{2} \mathrm{O}_{3}$ / $\left(\mathrm{Al}_{2} \mathrm{O}_{3}+\mathrm{CaO}^{*}+\mathrm{Na}_{2} \mathrm{O}+\mathrm{K}_{2} \mathrm{O}\right)^{*} 100$; molar concentrations) (Nesbitt et al., 1996). The CIA values (Table 3 ) vary from 95.2 to $98.9,87.9$ to 98.5 and 97 to 98.6 in LTI1, LTI2, and LTI3, respectively, indicating a high degree of weathering, and plot mostly towards the $\mathrm{Al}_{2} \mathrm{O}_{3}$ apex on the A-CN-K plot (Fig. 6b). This is further evaluated using the Mafic Index of Alteration (MIA $=100 \times\left[\left(\mathrm{Al}_{2} \mathrm{O}_{3}+\mathrm{Fe}_{2} \mathrm{O}_{3}(\mathrm{~T})\right) /\right.$ $\left(\mathrm{Al}_{2} \mathrm{O}_{3}+\mathrm{Fe}_{2} \mathrm{O}_{3}(\mathrm{~T})+\mathrm{MgO}+\mathrm{CaO}^{*}+\mathrm{Na}_{2} \mathrm{O}+\mathrm{K}_{2} \mathrm{O}\right.$ )) (Babechuk et al. (2014). MIA quantifies the net loss of the major mobile elements ( $\mathrm{Ca}, \mathrm{Mg}, \mathrm{Na}, \mathrm{K} \pm \mathrm{Fe}$ ) relative to the major immobile elements $(\mathrm{Al} \pm \mathrm{Fe})$. MIA values ranged from 91.2 to 99.8 in sediments (Table 3). The Index of Lateritization (IOL $=100 \times\left[\left(\mathrm{Al}_{2} \mathrm{O}_{3}+\mathrm{Fe}_{2} \mathrm{O}_{3}(\mathrm{~T})\right) /\right.$ $\left.\left(\mathrm{SiO}_{2}+\mathrm{Al}_{2} \mathrm{O}_{3}+\mathrm{Fe}_{2} \mathrm{O}_{3}(\mathrm{~T})\right)\right]$ ) (Babechuk et al., 2014) was used to delineate the state of advanced to extremely weathering. IOL values varies from 51 to 92,32-78, 75-94 in LTI1, LTI2, and LTI3, respectively (Table 3). The relationships between CIA, MIA and IOL is presented in Fig. 7, and indicate that both CIA and MIA are less varied between samples, in contrast to IOL, which varied widely between samples.

The $\mathrm{Fe} / \mathrm{Al}$ vs $\mathrm{Al}$ and $\mathrm{Al} / \mathrm{K}$ vs $\mathrm{Al}$ plots (Fig. 8) show the different contributions from catchment source laterites and soils; LTI1 and LTI3 are more closely related to Al-riched soils and lateritic materials while LTI2 is not. Furthermore, the discrimination functions F1 and F2 (Fig. 9a) were computed following the equation proposed by Roser and Korsch (1998). The discriminant functions are: F1 = $\left(-1.773 . \mathrm{TiO}_{2}\right)+\left(0.607 . \mathrm{Al}_{2} \mathrm{O}_{3}\right)+\left(0.760 . \mathrm{Fe}_{2} \mathrm{O}_{3}\right)+(-1.500 . \mathrm{MgO})+$ $(0.616 . \mathrm{CaO})+\left(0.509 . \mathrm{Na}_{2} \mathrm{O}\right)+\left(-1.224 . \mathrm{K}_{2} \mathrm{O}\right)+(-9.090) ; \mathrm{F} 2=$ $\left(0.445 . \mathrm{TiO}_{2}\right)+\left(0.070 . \mathrm{Al}_{2} \mathrm{O}_{3}\right)+\left(-0.250 . \mathrm{Fe}_{2} \mathrm{O}_{3}\right)+(-1.142 . \mathrm{MgO})+$ $(0.438 . \mathrm{CaO})+\left(1.475 . \mathrm{Na}_{2} \mathrm{O}\right)+\left(1.426 . \mathrm{K}_{2} \mathrm{O}\right)+(-6.861)$. These parameters distinguish the source of sediments into four provenance zone: P1, P2, P3, and P4. From the resulting F1 and F2 values (Fig. 9a), most sediment is grouped in the P3 field, indicating mafic characteristics.

\subsection{Characteristics of trace elements}

Trace element concentrations are listed in Table 1 . The distribution of the trace elements shows considerable variation and average trace element concentrations in the lake sediments decreases in the following order: $\mathrm{Zr}>\mathrm{V}>\mathrm{Cr}>\mathrm{Cu}>\mathrm{Zn}$ $>\mathrm{Ba}>\mathrm{Pb}>\mathrm{Ga}>\mathrm{Nb}>\mathrm{Th}>\mathrm{Y}>\mathrm{Sc}>\mathrm{Sr}>\mathrm{Ni}>\mathrm{Se}>\mathrm{Hf}>\mathrm{Mo}>\mathrm{Rb}$ $>\mathrm{As}>\mathrm{U}>\mathrm{Co}>\mathrm{Hg}$. In general, the lowest concentrations of these elements were observed in LTI2, while the highest concentrations were in LTI1. UCC normalized distribution patterns (Fig. 4) show that most of the elements are depleted in sediments, except $\mathrm{Se}, \mathrm{Hg}$ and $\mathrm{Cr}$. The main occurrence of the ferromangnesian elements $(\mathrm{Ni}$, $\mathrm{Cr}, \mathrm{V}$ and $\mathrm{Sc}$ ) and large cations ( $\mathrm{Y}, \mathrm{Nb}, \mathrm{Zr}$, Th and $\mathrm{Sr}$ ) show positive correlations with $\mathrm{Al}_{2} \mathrm{O}_{3}(\mathrm{r}=>0.65)$, reflecting association with clay fractions. Rb shows very high positive correlation with $\mathrm{K}(\mathrm{r}=0.97)$, reflecting control by K-bearing minerals. The positive correlation of $\mathrm{Sr}$ with $\mathrm{SiO}_{2}, \mathrm{Al}_{2} \mathrm{O}_{3}$, and $\mathrm{TiO}_{2}(\mathrm{r}=0.65-0.92)$ may suggest fixation 


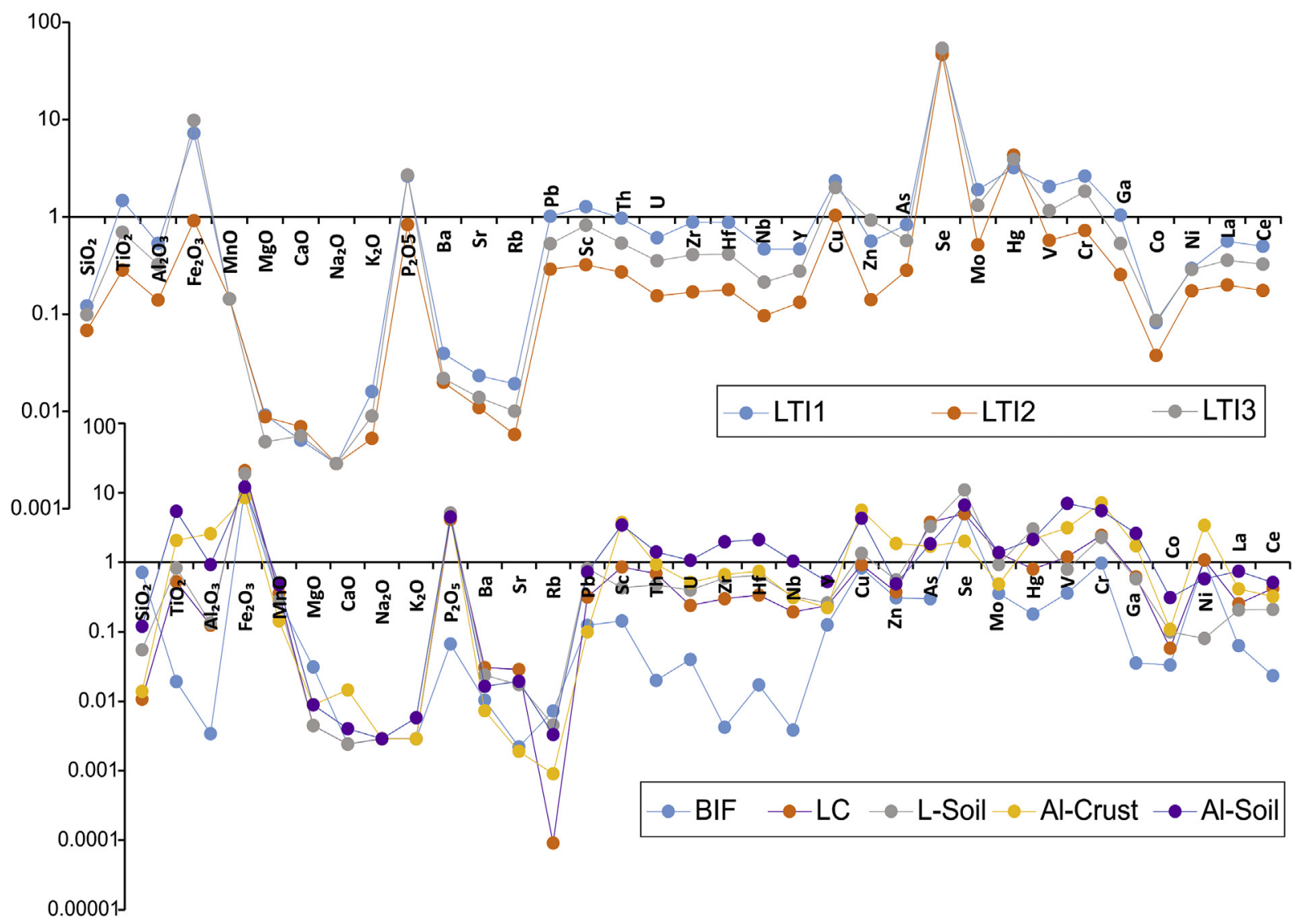

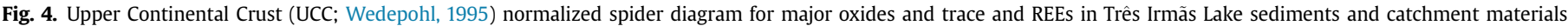
(lateritic crusts, LC; banded iron formation, BIF; Al-Crust; lateritic soil, L-Soil; and aluminous lateritic soil, Al-Soil).

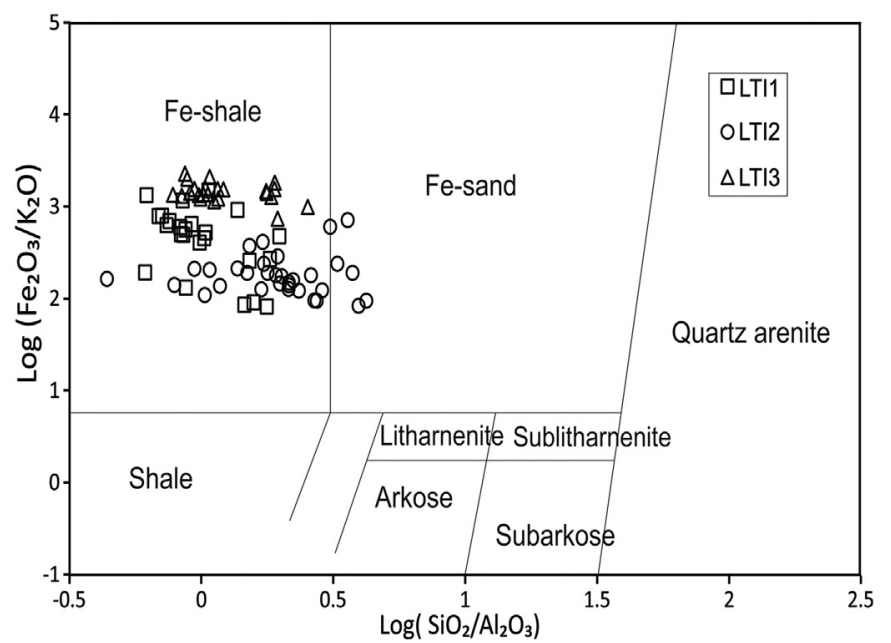

Fig. 5. (a) Geochemical classification of lake sediments based on $\log \left(\mathrm{SiO}_{2} / \mathrm{Al}_{2} \mathrm{O}_{3}\right)$ vs. $\log \left(\mathrm{Fe}_{2} \mathrm{O}_{3} / \mathrm{K}_{2} \mathrm{O}\right)$ (after Herron, 1988).

by aluminosilicate clay minerals. A strong positive correlation $(\mathrm{r}=0.99)$ between $\mathrm{Zr}$ and $\mathrm{Hf}$ with $\mathrm{SiO}_{2}$ indicates the influence of heavy minerals. The positive correlation between $U$ and Th with $\mathrm{Al}_{2} \mathrm{O}_{3}$ and $\mathrm{TiO}_{2}$ suggests $\mathrm{Al}$-silicates hosting. A significant positive correlation among the $\mathrm{REE}, \mathrm{TiO}_{2}$ and $\mathrm{Zr}$ suggests that these elements are contained in zircon or maybe anatase (Barros et al., 2005). In general, the occurrence of elemental groups with any potential host as outlined above is consistent with typical geochemical behavior.

The Th/Sc vs. Zr/Sc plot (Fig. 10) indicates the degree of sorting and/or recycling effect of sediments. The elemental ratios $\mathrm{Fe} / \mathrm{Al}, \mathrm{Zr} / \mathrm{Hf}$, $\mathrm{Al} / \mathrm{Th}, \mathrm{Ti} / \mathrm{Zr}, \mathrm{Hf} / \mathrm{Nb}, \mathrm{Co} / \mathrm{Th}, \mathrm{Ba} / \mathrm{Sr}, \mathrm{La} / \mathrm{Gd}, \mathrm{La} / \mathrm{Al}$, and $\mathrm{Zr} / \mathrm{Y}$ in the sediments were within a small range in all lakes (Table 3 ) and most of these ratios show a nearly similar range for catchment lateritic crusts and soils but are different from BIF. The binary plot of $\mathrm{La} / \mathrm{Th}$ and $\mathrm{Th} / \mathrm{Yb}$ (Fig. 10) indicates that most of the lake sediments have a mafic source. Trace element occurrence can also be used to infer the redox condition in the epositional sedimentary environment (Tribovillard et al., 2006): $\mathrm{V} / \mathrm{Cr}$ ratios $<2$ indicate an oxic environment; $2-4.5$, dysoxic conditions and $>4.5$ anoxic environment (Jones and Manning, 1994). The average $\mathrm{V} / \mathrm{Cr}$ ratios (Table 3 ) of the studied sediments are 1.2,1.25 and 0.97 in LTI1, LTI2, and LTI3, respectively, suggesting the sediments were deposited in an oxic environment.

\subsection{Rare earth elements (REEs)}

The REE concentrations varied considerably in the different lake sediments, with $\Sigma$ REE being highest in LTI1 $(21.8-121 \mathrm{mg} / \mathrm{kg})$, followed by LTI3 (35-65 mg/kg) and LTI2 (13-49 mg/kg) (Table 1); all are relatively depleted compared to UCC. The REEs were divided into two conventionally termed subgroups: (i) light REE (LREE, La through Eu), and (ii) heavy REE (HREE, Gd through Lu). LREE, 

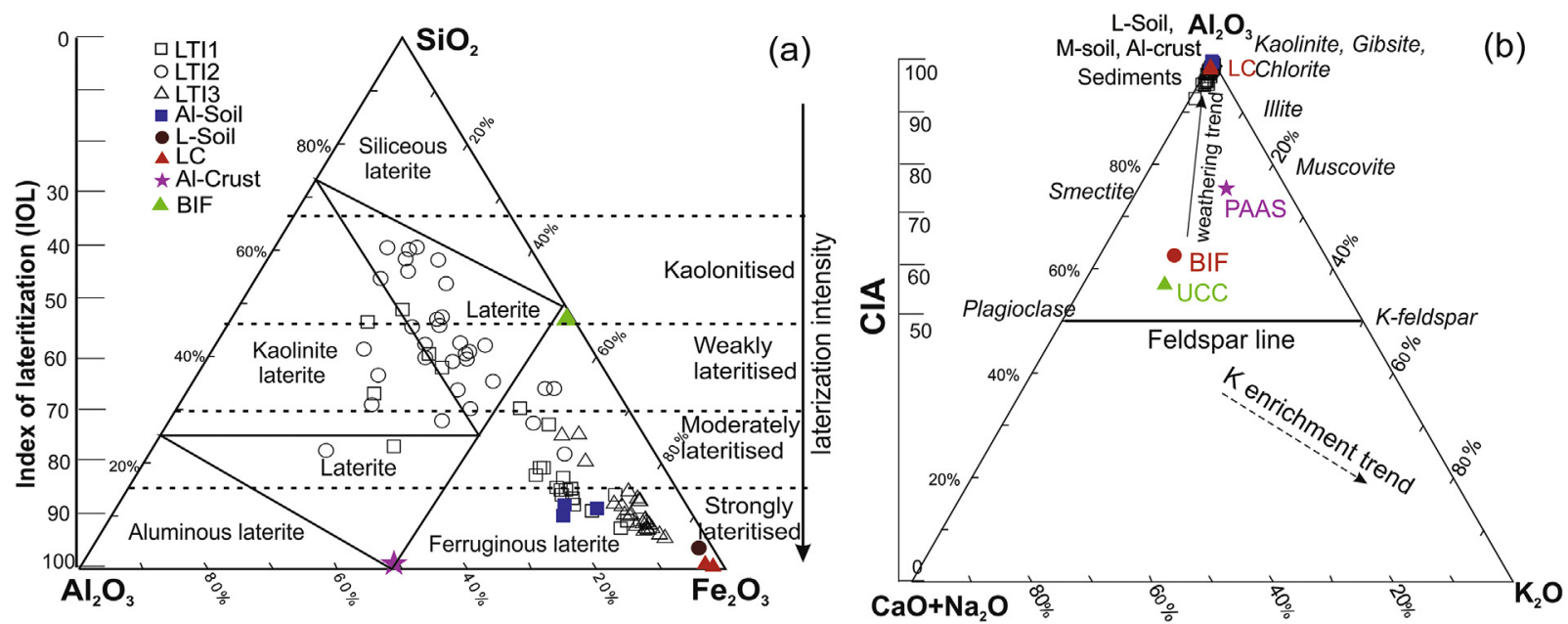

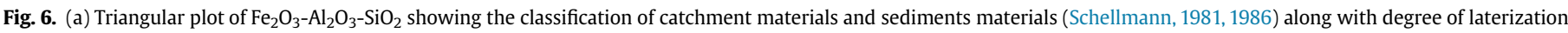

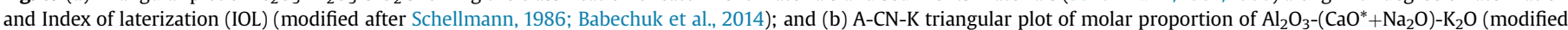

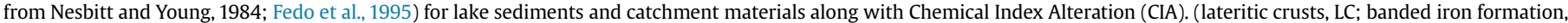
BIF; Al-Crust; lateritic soil, L-Soil; and aluminous lateritic soil, Al-Soil).

especially La and $\mathrm{Ce}$, are more abundant than heavy REE (HREE). Good positive correlation between $\mathrm{Al}_{2} \mathrm{O}_{3}$ and the $\mathrm{REE}(\mathrm{r}=0.95)$ indicates that they are mostly concentrated in the Al-silicate fractions (clay). $\mathrm{La}_{\mathrm{N}} / \mathrm{Sm}_{\mathrm{N}}$ (3.3-7.6) fairly consistent through all the samples (Table 3), indicating that LREEs are not significantly fractionated during weathering and pedogenesis process. Similarly, Gd/ $\mathrm{Yb}$ varies from 0.87 to 2.76, showing HREE is not fractionated in sediments. Fractionation of Eu can be tracked using the Eu anomaly $\left[\mathrm{Eu} / \mathrm{Eu}^{*}=\mathrm{Eu}_{\mathrm{N}} /\right.$ square-root $\left(\mathrm{Sm}_{\mathrm{N}} \mathrm{x} \mathrm{Gd}_{\mathrm{N}}\right.$; $\mathrm{N}$ : normalized to chondrite values; Schmitt et al., 1963]. In the sediments, the Eu anomaly varies from 0.63 to $0.85,0.5-0.9$ and $0.65-0.86$ for LTI1, LTI2, and LTI3, respectively, indicating a similar and less pronounced Eu occurrence. This is closer to UCC (0.69), laterites (0.62 - 0.9), and soils $(0.81-0.97)$, but very different from BIF (4.26). The poor correlation between $\mathrm{CIA}$ and $\mathrm{Eu} / \mathrm{Eu}^{*}(\mathrm{r}=<0.1)$ indicates that $\mathrm{Eu}$ is poorly correlated with weathering intensity. The Ce anomaly (Ce/ $\mathrm{Ce}^{*}=\mathrm{Ce}_{\mathrm{N}} /\left(\right.$ square-root $\left.\left(\operatorname{La}_{\mathrm{N}}{ }^{*} \operatorname{Pr}_{\mathrm{N}}\right)\right)$; $\mathrm{N}_{\mathrm{N}}$ : normalized to chondrite values) in the sediments is $2-4.5,1.8-3.59$, and $3.3-3.97$ for LTI1, LTI2, and LTI3, respectively. The chondrite normalized REE distribution patterns (Fig. 11) suggest that lake sediments have similar REE distributions characterized by LREE enrichment, and mostly flat HREEs and lack of an Eu anomaly. Chondrite normalized REEs are similar to that displayed by laterite crust, except for the Ce anomaly, and soils, while relatively different than BIF, UCC and PAAS.

\subsection{Principal Component Analysis (PCA)}

Two principal components (PC) were extracted (Fig. 12), with Eigen values $>1$, which taken together represent $79.47 \%$ (PC1 77.8\%; PC2 6.9\%) of the total variance. Based on the weighting of the contribution of each variable, there is high positive loading for $\mathrm{Al}, \mathrm{Ti}$, $\mathrm{P}, \mathrm{Ba}, \mathrm{Sr}, \mathrm{Pb}, \mathrm{Sc}, \mathrm{U}, \mathrm{Th}, \mathrm{Nb}, \mathrm{Y}, \mathrm{Cu}, \mathrm{Zn}, \mathrm{Mo}, \mathrm{V}, \mathrm{Cr}, \mathrm{Ga}$, LREE and HREE (Group-1) and high negative loading for TOC, Hg and TOS (Group-3) in PC1. PC 2 is characterized by high positive loading of $\mathrm{Fe}_{2} \mathrm{O}_{3}$, As and Zn (Group 2). In general, Group 1 elements represent terrigenous input influenced by Al-clays and are mainly dominate in LTI1, Group2 are influenced by Fe-oxyhydroxides and dominate in LTI3, and Group- 3 is attributed to the biogenic fraction and mainly dominate in TLI2.

\section{Discussion}

\subsection{Factor controlling distribution of organic matter in surface} sediments

Although the possibility of nitrogen isotope be affected by inorganic nitrogen, our interpretation is based on the integration of both isotopes and elemental ratios. Additionally, the absence of terrestrial derived inorganic nitrogen in the analyzed samples may be supported by the analysis of TN versus TOC (Castro et al., 2010). Thus, $\rho>0.9$ between TN and TOC of sedimentary organic matter in this study suggests that nitrogen did not derive from external inorganic sources.

The vegetation types of the Três Irmãs Lake basin are mainly characterized by $C_{3}$ plants from forest formation and canga vegetation (Fig. 3; Table A1), which could account for lower values of $\delta^{15} \mathrm{~N}$ and C/N in lake sediments (Tyson, 1995; Meyers, 1994; Sahoo et al., 2015, 2016). However, sediments of LTI1 and LTI3, with relatively enriched $\delta^{15} \mathrm{~N}$, probably reflect the contribution of organic matter from algae and macrophytes. Since these were only observed in the margins of LTI1, we can assume that algae may be a predominant component for the western and shallower margin of LTI3, near the connection with LTI2. Lower values of $\delta^{15} \mathrm{~N}$ in sediments of LTI2 may indicate a higher contribution from $C_{3}$ vascular plants in the catchment basin, represented by canga vegetation. LTI 2 contains the highest TOC among all the lakes. Higher TOC with lower $\delta^{15} \mathrm{~N}$ values suggest that sediments of LTI2 may be similar to swamps (filled lakes) of the Serra Sul dos Carajás (e.g. Sahoo et al., 2016). These finding are supported by the fact that canga vegetation (allochthonous source) do not provide enough organic detritus to be transported by surface runoff until the central basin of the lake (Sahoo et al., 2015, 2016). Therefore, only an autochthonous source may explain these elemental and isotopic signals for an active lake such as LTI2. This suggests that this lake was a former swamp that may have evolved into an active lake following an increase of the accommodation space by subsidence or a rise in water levels. Further studies using radiometric dating could delineate how the lake evolved (filled) over time. 


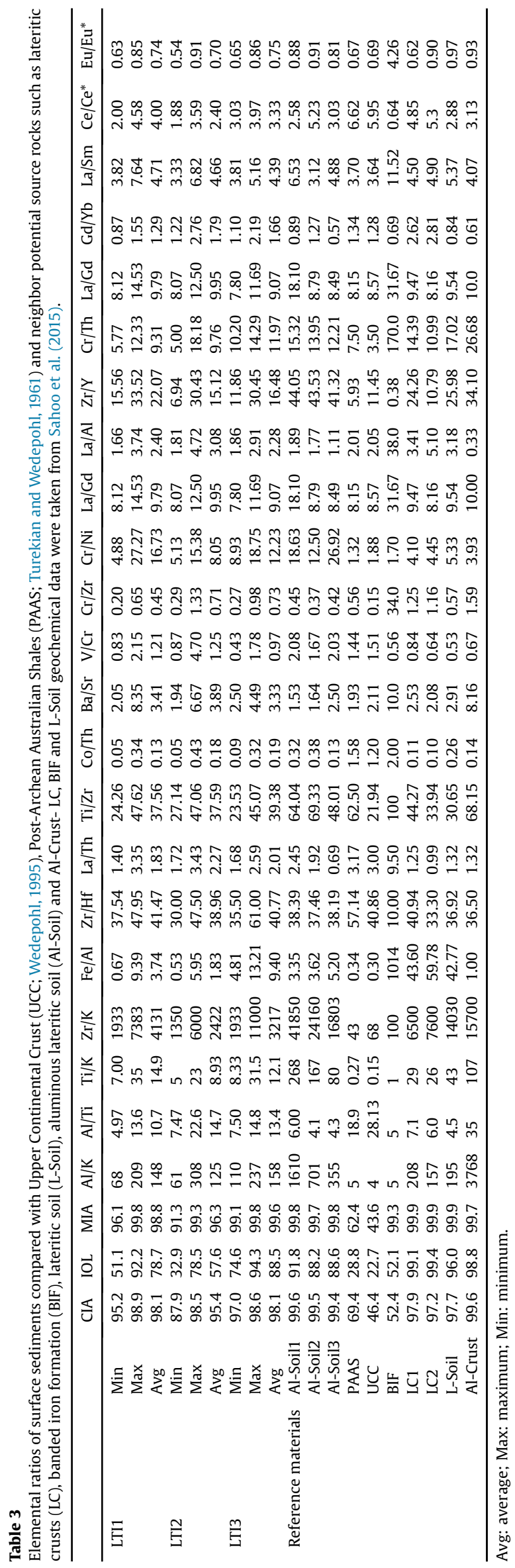

\subsection{Factor controlling distribution of inorganic components in surface sediments}

The elemental composition of sediments is influenced by several factors, including lithological composition of the provenance area, chemical weathering, diagenesis and depositional and post-depositional process (Condie, 1991; Sahoo et al., 2016). Based on the distribution of elements and sedimentary facies, there is a clear differentiation among the three lakes. TOC is predominant in all LTI2 samples, while major detrital elements like Fe and $\mathrm{Al}$ are dominant in LTI1 and LTI3. This apparent spatial variability is probably the result of a difference in their catchment geology and drainage pattern as well as lake morphology and morphometry, which controls the lake productivity and distribution of allochthonous and autochthonous OM over the lake basin. $\mathrm{Al}$ and $\mathrm{Fe}$ are relatively immobile during laterization. Thus, the $\mathrm{Fe} / \mathrm{Al}$ ratios in sediments can be a key to the origin of different source rocks (Dezileau et al., 2007; Pattan et al., 2012). On Fe/Al vs Al and Al/K vs Al plots (Fig. 8), LTI1 is more influenced by alumnous lateritic soils, while LTI3 is influenced by both alumnous lateritic and lateritic soils. However, less contribution of these materials were noted for LTI2. This interpretation correlates well with the catchment lithology, and shows that catchment lithology is one of the important factors controlling distribution of inorganic fractions in the basin.

Furthermore, PCA (Fig. 12) shows high positive loading of most of the trace elements, REEs, $\mathrm{Al}, \mathrm{K}$, and Ti in Group 1, while these elements are poorly correlated with Fe (Group 2) and TOC (Group 3 ). Strong positive correlation of most of the trace and REEs with $\mathrm{Al}_{2} \mathrm{O}_{3}$ and $\mathrm{TiO}_{2}$ in Group 1 indicates they are likely scavenged by aluminosilicate minerals (Marker et al., 1991). Based on the distribution of $\mathrm{Al}_{2} \mathrm{O}_{3}$, these elements are likely enriched in LTI1, mainly in the central part of the lake, towards the eastern border. These elements represent the original lithology prior to weathering and erosion of catchment rocks and soils. Enrichment of this fraction close to the northeast part of LTI1, as well as in catchment mafic soils, indicates that mechanical erosion was important in the catchment area and responsible for deposition at the margin and center of the lake. Although $\mathrm{Fe}$ is terrigenous, the difference of distribution of Fe relative to other detrital elements such as $\mathrm{Al}$ and Ti suggests a different chemical behavior during weathering and transport, because Ti and Al exhibit low mobility, the opposite of Fe, which has comparatively high mobility. Also, the distribution of Fe in lake surface could be influenced by catchment drainage patterns, soil availability, and/or bottom topography.

A strong positive $\mathrm{Zr}$ and $\mathrm{Hf}$ correlation indicates presence of heavy minerals, such as zircon might be controlling their distribution in the sediments. Moreover, a $\mathrm{Zr} / \mathrm{Hf}$ ratio close to 40 fits the zircon variation interval (Barros et al., 2005). The positive correlation between $\mathrm{Zr}$ and REE indicates their preference for zircon. However, Ti is also positively correlated with REE, the same elements that correlates to $\mathrm{Zr}$, suggesting that both zircon and anatase have accumulated together in the lateritic profile (Santos et al., 2016). The very similar distribution of $\mathrm{Zr}$ and $\mathrm{SiO}_{2}$ in the SW portion of the LTI1 and the decrease in their concentrations towards the center of the lake are probably a function of hydraulic sorting of heavy minerals. In general, geochemical differences among sediment textures can be attributed to separation by hydraulic sorting, which is controlled by the source to sink distance, energy of the transport system, and other factors (Roy and Roser, 2012). Hydraulic sorting of minerals can also control the distribution of Th, $\mathrm{U}$, $\mathrm{Zr}$, Hf, and $\mathrm{Nb}$ in clastic sediments (Armstrong-Altrin, 2004). The relationship between $\mathrm{Th} / \mathrm{Sc}$ and $\mathrm{Zr} / \mathrm{Sc}$ (McLennan et al., 1993) was used to evaluate the $\mathrm{Zr}$ enrichment due to sorting and/or recycling effect. On Th/Sc and $\mathrm{Zr} / \mathrm{Sc}$ plot (Fig. 10), Zr enrichment is shown by 

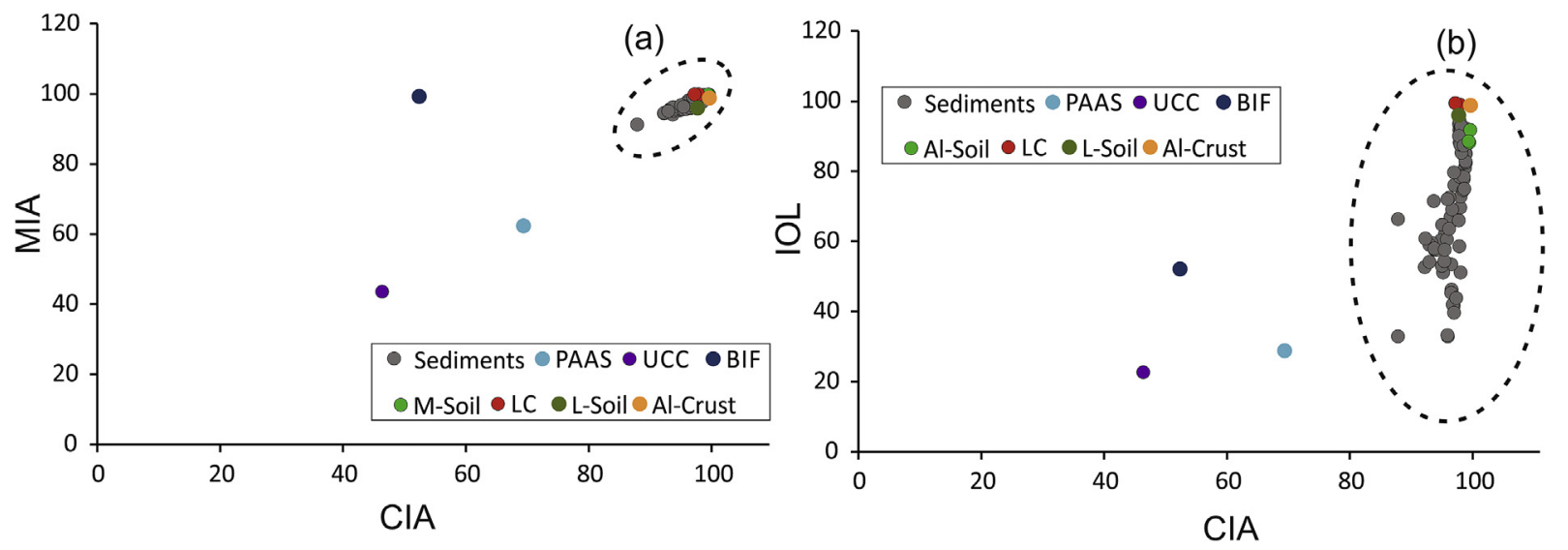

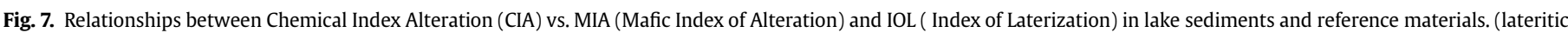
crusts, LC; banded iron formation, BIF; Al-Crust; lateritic soil, L-Soil; and aluminous lateritic soil, Al-Soil).

the gradual increase in the $\mathrm{Zr} / \mathrm{Sc}$ ratio and accompanying deviation from the compositional trend. This indicates zircon enrichment during selective sorting and reworking processes in the sediment evolution (McLennan et al., 1993). However, similar Zr enrichment in catchment LC and soils samples also indicates the influence of source materials.

Redox processes accompany OM mineralization and early diagenesis favors precipitation and/or dissolution of $\mathrm{Fe}-$ oxydroxides and formation of authigenic of Fe-carbonates (such as siderite) and sulfides (such as pyrite) (Tapia and Audry, 2013), which can control the redistribution of trace and REE (Quinn et al., 2006; Pourret and Davranche, 2013; Och et al., 2014; Vermeire et al., 2016). Siderite formation is expected in Fe and organic rich lacustrine sediments and previous work has reported siderite formation in Carajás lakes (Soubies et al., 1991; Costa et al., 2005; Hermanowski et al., 2012). However, in the Três Irmãs Lake basin this is unlikely in the surface sediments $(<10 \mathrm{~cm})$ due to prevalence of oxic conditions, as indicated by the positive correlation between Fe and As $(\mathrm{r}=0.8)$ and $\mathrm{V} / \mathrm{Cr}$ ratios $(<2)$ in surface sediments. Also, sulfide formation is unlikely due to very low concentrations of $\mathrm{S}$ and the poor correlation between Fe and S in sediments. Significant positive correlation of most of the trace and REEs with $\mathrm{Al}(\mathrm{r}>0.7)$, $\mathrm{Ti}(\mathrm{r}>0.8)$, and/or Zr compared to low correlation with $\mathrm{Fe}(\mathrm{r}=0.02)$ and TOC $(r=0.03)$ suggest that they were not significantly fractionated during post-depositional diagenesis.

The sediments are geochemically classified as Fe shale type (Fig. 5), which indicates a strong influence from catchment laterites. Laterite is commonly formed by the decomposition of the source rock under different stages of chemical weathering (El-Leil et al., 2017), which leads a progressive change in the ratio of Si and sesquioxide. Hence the ratio of $\mathrm{SiO}_{2}, \mathrm{Fe}_{2} \mathrm{O}_{3}$ and $\mathrm{Al}_{2} \mathrm{O}_{3}$ is define the extent of laterization (Schellmann, 1981). According to Karunakaran and Sinha Roy (1981), these catchment laterites are classified as ferruginous laterites since their $\mathrm{Fe}_{2} \mathrm{O}_{3}: \mathrm{Al}_{2} \mathrm{O}_{3}>1$ and $\mathrm{SiO}_{2}: \mathrm{Fe}_{2} \mathrm{O}_{3}<1.33$. Similar classification is based on the $\mathrm{Al}_{2} \mathrm{O}_{3}-\mathrm{SiO}_{2}-$ $\mathrm{Fe}_{2} \mathrm{O}_{3}$ ternary diagram (Fig. 6a), indicating that the catchment laterites are formed during strong laterization. The A-CN-K diagram (Fig. 6b) also indicates the formation of lateritic rocks as a consequence of strong chemical weathering that resulted in plagioclase and K-feldspar dissolution and the concomitant depletion of $\mathrm{Ca}, \mathrm{Na}$, and $\mathrm{K}$ relative to $\mathrm{Al}$, which was retained within pedogenetic clays (Nesbitt and Young, 1984; Babechuk et al., 2014). This is consistent with the CIA (Fig. 6b). As pointed out by Fedo et al. (1995) the source materials can be reconstructed if the studied weathering trend is extrapolated backwards to the plagioclase and K-feldspar connecting line in the A-CN-K diagram. However, no weathering trend was observed here as both sediments and catchment materials plot near the $\mathrm{Al}_{2} \mathrm{O}_{3}$ apex with little variation of CIA. Thus, the $\mathrm{CIA}$ is ineffective at quantifying or differentiating elemental changes during the advanced stages of weathering.

This is further evaluated using the new mafic index of alteration (MIA) proposed by Babechuk et al. (2014). The index extends the equation of the CIA to include the mafic elements $\mathrm{Mg}$ and Fe. Iron, in general, is enriched along with Al by the formation of highly insoluble Fe-oxyhydroxides in oxidative weathering environments (e.g., Driese, 2004). Increasing MIA values represent progressively more weathered rock, and a value of 100 indicates complete loss of the mobile elements. However, MIA values are high and relatively invariable (from 91 to 99), similar to the CIA, and both are highly linearly correlated (Fig. 7a) showing that the MIA suffers from the same inability to quantify the advanced to extreme stages of weathering. During lateritization the dominant process is desilication but $\mathrm{Si}$ is not considered into the CIA. Keeping this in mind, Babechuk et al. (2014) proposed a different approach, the Index of Lateritization (IOL) using the mass (wt. \%) ratio of $\mathrm{SiO}_{2}, \mathrm{Fe}_{2} \mathrm{O}_{3}$, and $\mathrm{Al}_{2} \mathrm{O}_{3}$, as shown in Section 4.3. The IOL value is designed to directly accompany the $\mathrm{SiO}_{2}-\mathrm{Al}_{2} \mathrm{O}_{3}-\mathrm{Fe}_{2} \mathrm{O}_{3}$ ternary diagram, similar to the approach of Hill et al. (2000). Unweathered mafic rocks, in general, have IOL values $<40$ and higher IOL values correspond to more intensely weathered samples (Babechuk et al., 2014; reference therein). The calculated IOL values varied widely, unlikely as CIA and MIA (Fig. 7b), for catchment laterites fall within strong lateritization, but the weathered sediments fall between the strong to weak field divisions and a few are in the kaolinitised field (Fig. 6a), indicating chemical reworking of sediments. Higher IOL values are consistent with the increase in Fe concentrations in the sediments. Therefore, comparisons with the IOL may help to identify samples are extremely weathered and reworked samples that do not meet the criteria for strict in situ formation.

Laterization controls the enrichment and distribution of major and trace elements in laterites (El-Leil et al., 2017) and resulted in very concentrations of mobile elements such as $\mathrm{Na}, \mathrm{K}, \mathrm{Mg}$, Ca and $\mathrm{Si}$ and relative enrichment of Fe and Al. However, high enrichment of Fe relative to $\mathrm{Al}$ can be influenced by rock types, e.g., quartz-bearing rocks. In this case, Fe is relatively enriched because some quantities of Al are dissolved during desilication (Schellmann, 1981). Also, the mobility of Fe and Al are highly influenced by $\mathrm{pH}$ and Eh (Lelong et al., 1976). These factors lead to separation of Fe and $\mathrm{Al}$ and formation of secondary minerals which control the chemical behavior of trace elements in laterites under different environmental 


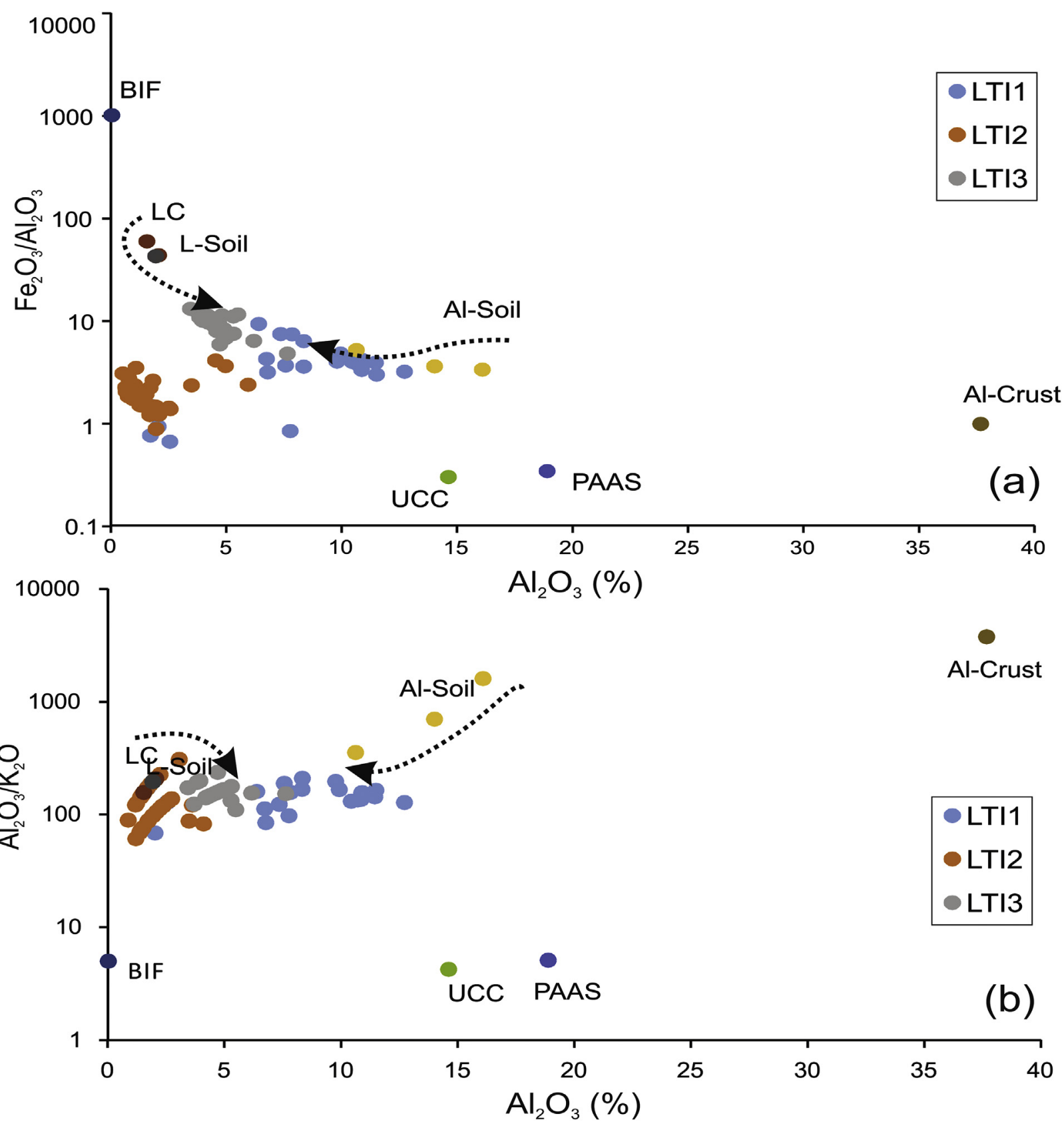

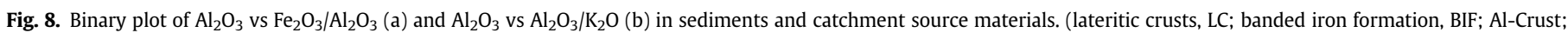
lateritic soil, L-Soil; and aluminous lateritic soil, Al-Soil).

conditions (Lelong et al., 1976; El-Leil et al., 2017). Strong occurrence of $\mathrm{Al}$ and $\mathrm{Ti}$ with and most of the trace elements, including REEs, in sediments may be indicative of silicates minerals alteration during early weathering stages of laterization followed by recombination of these elements within the crystal structure of aluminosilicate minerals (Marker et al., 1991). As thus, these elements had low reactivity during deposition and post-deposition of sediments. Also, lake sediments have lower LREE and HREE fractionations, indicating they are less altered during chemical weathering. In addition, the lack of correlation of $\mathrm{Ce} / \mathrm{Ce}^{*}$ and $\mathrm{Eu} / \mathrm{Eu}^{*}$ with any of the weathering indices also indicates that $\mathrm{Eu}$ and $\mathrm{Ce}$ losses are poorly correlated with weathering intensity. Low Mn could be due to mobilization of $\mathrm{Mn}$ under low $\mathrm{pH}$ and high $\mathrm{CO}_{2}$ activities (Lelong et al., 1976).

\subsection{Source of inorganic components in surface sediments}

Similar geochemical behavior between sediment and catchment laterite crust and soil in the $\mathrm{A}-\mathrm{CN}-\mathrm{K}$ plot indicates lateritic crust weathering and pedogenesis that allows degradation (deferruginisation) of duricrusts into a friable and soft matrix in a progressively weathering process to form soils (Horbe and Costa, 2005), which are the major inorganic source of lake sediments. As a consequence, the geochemical ratios of $\mathrm{Zr} / \mathrm{Hf}, \mathrm{Al} / \mathrm{Th}, \mathrm{Ti} / \mathrm{Zr}, \mathrm{Hf} / \mathrm{Nb}$, $\mathrm{Co} / \mathrm{Th}, \mathrm{Ba} / \mathrm{Sr}, \mathrm{La} / \mathrm{Gd}, \mathrm{La} / \mathrm{Al}, \mathrm{Zr} / \mathrm{Y}$ and $\mathrm{Eu} / \mathrm{Eu}^{*}$ in sediments maintained similar to those of catchment lateritic crusts and soils. The chondrite-normalized REE patterns for sediment were very similar for all three lakes; enrichment of LREE, flat HREE with lack of an Eu anomaly. This pattern is similar to the catchment laterite crusts and 

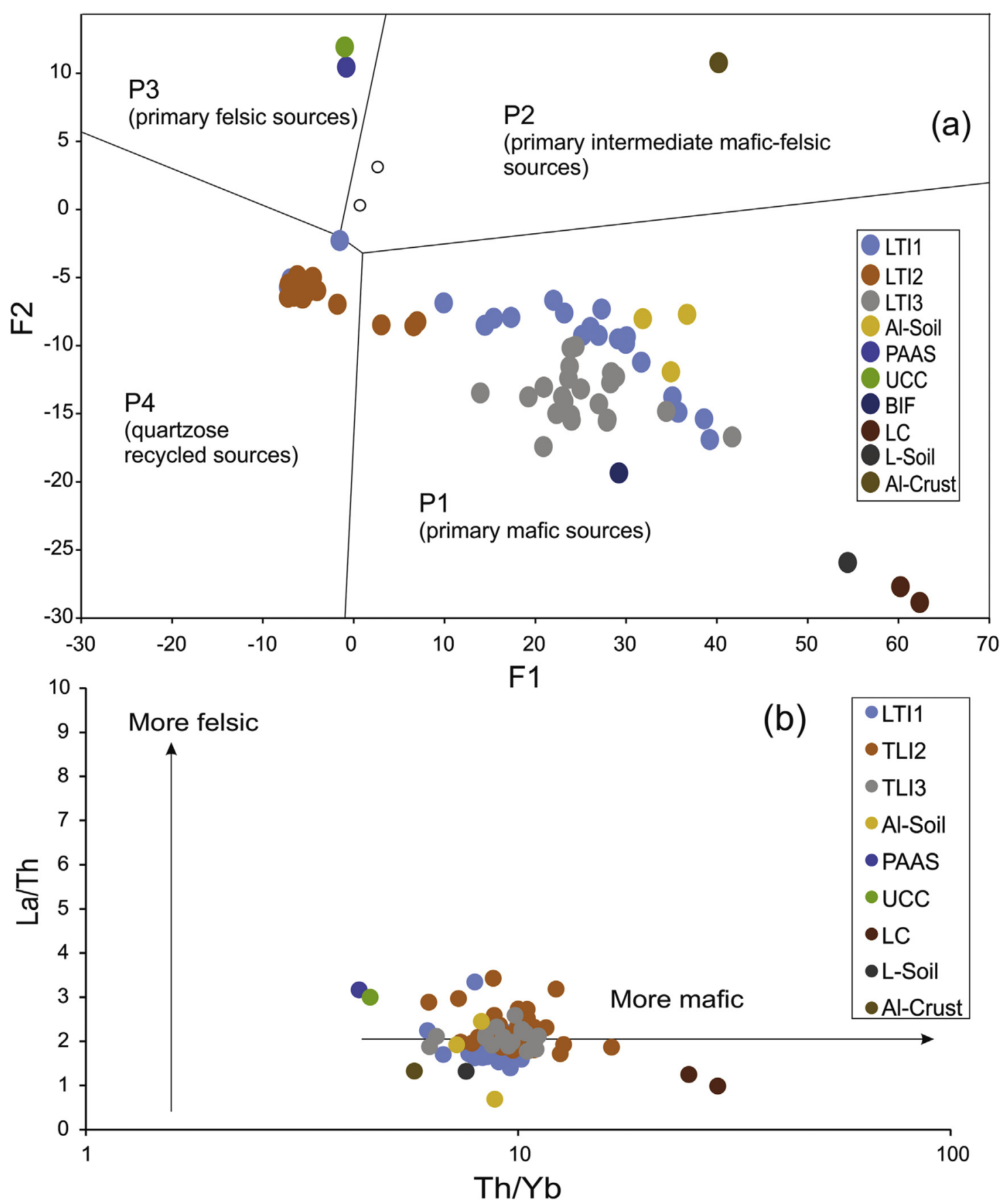

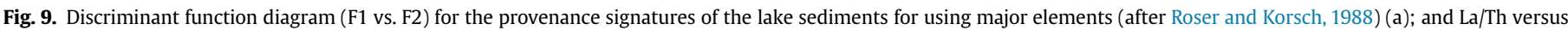

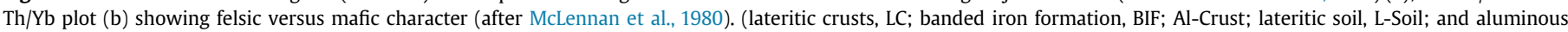
lateritic soil, Al-Soil).

soils, except the positive Ce anomaly which can be interpreted as scavenging $\mathrm{REE}^{3+}$ (except for $\mathrm{Ce}^{4+}$ ) from the laterites under oxidizing conditions (Sanematsu et al., 2011), further reinforceing that the geochemical analogies between the lake sediments and the lateritic crust and soils are similar.

$\mathrm{Al}$ and Fe are relatively immobile during laterization. Thus, the $\mathrm{Fe} / \mathrm{Al}$ and $\mathrm{Al} / \mathrm{K}$ ratios in sediments can be a key factor for understanding the contribution from different catchment rocks. Fe/Al vs $\mathrm{Al}$ and $\mathrm{Al} / \mathrm{K}$ vs $\mathrm{Al}$ plots (Fig. 8) shown that laterite crust has significant influence on all three lakes (though comparatively lower on LTI2), while Al-enriched soil has more influence on LTI1.
However, relatively low $\mathrm{Fe} / \mathrm{Al}$ ratios in sediments compared to laterite crust is due to increase in $\mathrm{Al}$ in sediments via progressive transformation of crust into soils and mixing with aluminous laterite soil. This transformation altered the concentration of major elements; however, it did not modify the trace element ratios and REE patterns. Therefore, during this process, the sediments maintained the same geochemical signature as the lateritic crust. Although, the Al-Crust does not seem to have had any direct influence on lake sediments, we cannot ignore its contribution because it could be the main source rock for aluminous lateritic soils in the catchment. Overall, the sediment compositions 


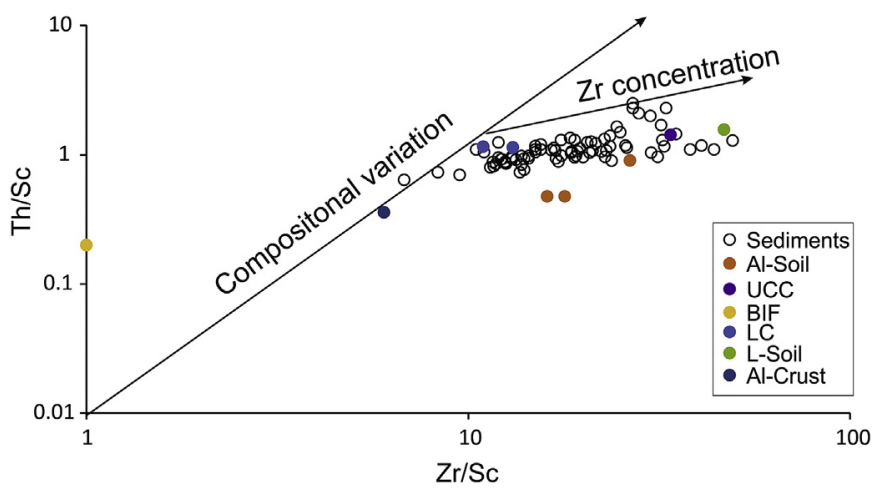

Fig. 10. Binary plot of Th/Sc vs. Zr/Sc (after McLennan et al., 1993) for the Três Irmâs lake sediments. The concentration of zircon due to sediment sorting and recycling can be seen along Trend 2. (Upper Continental Crust, UCC; lateritic crusts, LC; banded iron formation, BIF; Al-Crust; lateritic soil, L-Soil; and aluminous lateritic soil, Al-Soil).

correlate well with the catchment lithology indicating that catchment lithology is one of the important factors controlling distribution of inorganic fractions in lake sediments.

Laterites are the residual products developed on a large variety of parent rocks under intense chemical weathering conditions. It is thus important to understand the type of source rocks from which the laterites formed as well as contribution of other rocks that control the detritic composition of lake sediments. The geology of this region shows the existence of BIF, and other metavolcanic and mafic rocks around the Carajás basin (Gibbs et al., 1986; Teixeira and Eggler, 1994; Olszewski et al., 1989). Laterite crust is mainly related to BIF. However, our geochemical results indicate that the catchment laterite crust is significantly different from BIF, as shown by trace elements ratios and REE behavior. This could be due to the fact that lateritic crust had a subordinate influence relative to regionally available rocks. Fe enrichment in laterites suggests ironrich basic parent rocks as they contain more mafic minerals with higher amounts of ferrous iron than in the acid rocks (Sayyed, 2014). Hayashi et al. (1997) demonstrated that $\mathrm{Al} / \mathrm{Ti}$ ratios for provenance discrimination, with ranges from 3 to 11 for mafic rocks, $11-21$ for intermediate rocks and $21-70$ for felsic rocks. The average $\mathrm{Al} / \mathrm{Ti}$ ratios of the studied sediments samples for the Três Irmâs lakes varies range from 4.9 to $12.5,8.9-21$ and 7.5-15 in LTI1, LTI2, and LTI3, respectively, suggesting their derivation from intermediate to mafic rock. This is further supported using the discrimination diagram (Fig. 9a), which shows that most sediments are grouped in the P3 field, indicating the source rock could have mafic characteristics.

Selected immobile trace elements such as $\mathrm{Th}, \mathrm{Zr}, \mathrm{Hr}, \mathrm{Ni}$, and $\mathrm{Cr}$ and REEs can also be used as a potential tool to characterize the provenance of clastic sediments (Cullers, 1995; Das and Haake, 2003). The concentrations of $\mathrm{Th}, \mathrm{Zr}$, Hf and REEs in general are enriched in felsic rocks relative to mafic rocks because they are highly incompatible during most igneous melting and fractionation processes (Cullers, 1995; McLennan, 1989). Depletion of these elements in sediments implies a felsic source is less significant. La/Th vs Th/Yb (Fig. 9b) also indicates mafic contribution of the sediments (McLennan et al., 1980). Additionally, the REE patterns may differ with respect to sources (Cullers, 1995; McLenna et al., 1993). Felsic rocks commonly favor with strong differentiation of LREEs from HREEs and striking negative Eu anomaly, whereas the lack of, or a small Eu anomaly, is usually related to input from mafic detritus, and large anomalies are related to felsic sources (McLennan et al., 1993; Cullers, 2000). The lack of an Eu anomaly in our sediments further suggests a relatively mafic source rock.

\section{Conclusions}

In this study, we investigated the elemental geochemistry of

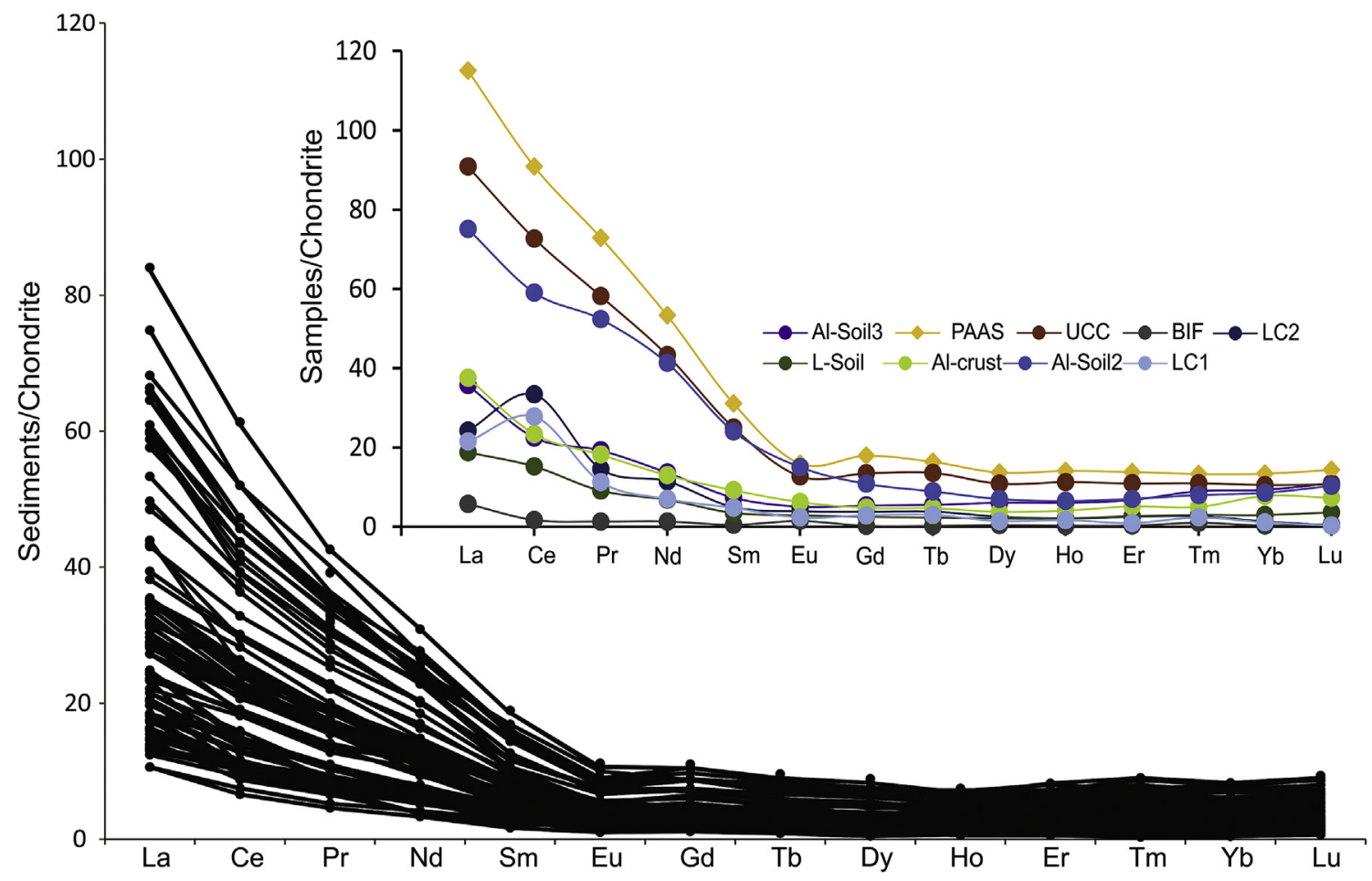

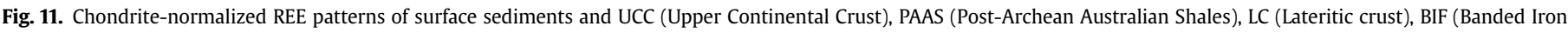
Formation), lateritic soil (L-Soil) and aluminous lateritic soil (Al-Soil). 


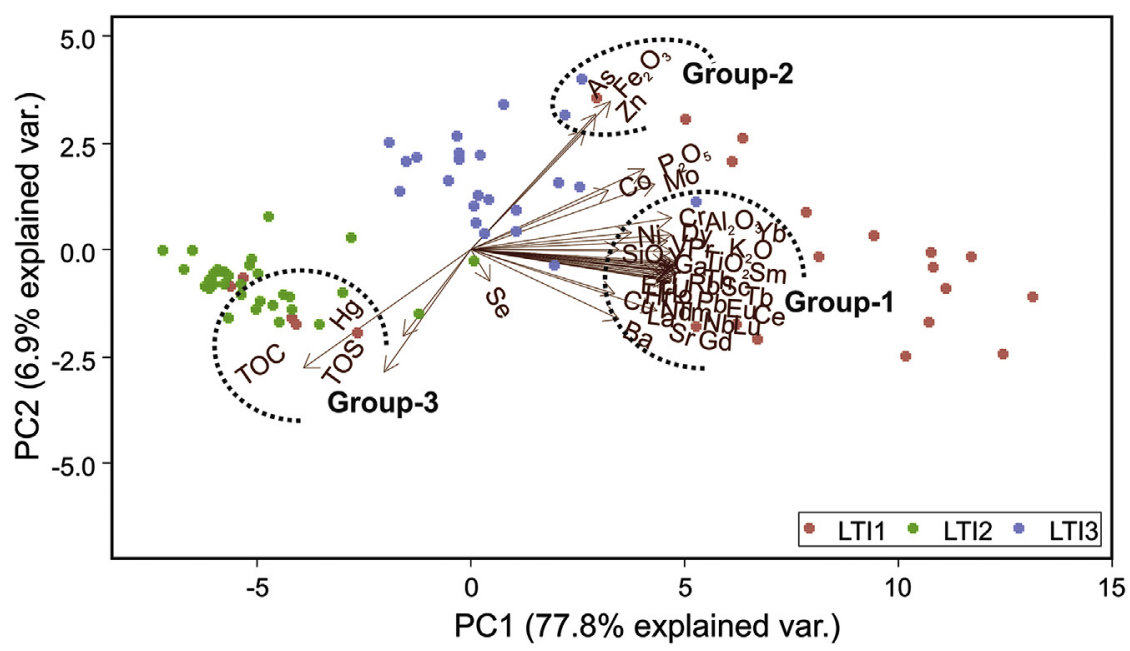

Fig. 12. Principal component loading plot of PC1 vs PC2 extracted from the Factor Analysis of the geochemical data of all Três Irmâs lake sediments.

Lake Três Irmãs sediments, and the following conclusions were obtained:

- The studied sediments are enriched with $\mathrm{Fe}_{2} \mathrm{O}_{3}, \mathrm{P}_{2} \mathrm{O}_{5}$ and $\mathrm{Se}$ relative to UCC and depleted in other major oxides and trace elements and their spatial distribution is mainly influenced by catchment lithology, weathering processes and elemental mobility.

- Organic matter in the lake sediments is derived from terrestrial plants, macrophytes and freshwater DOC. However, LTI2 has a similar signal to swamps (filled lakes) within the studied plateau.

- The CIA, and MIA values suggest that high chemical weathering at source areas and for these sediments; however, Index of Lateritization (IOL) is a more reliable evaluation of weathering intensity and can provide additional insight into weathering transformations.

- Trace and REEs in the sediments are hosted in aluminosilicate phases during laterite weathering.

- Sediments were deposited under oxidized conditions, and sorting and post-depositional weathering and diagenetic changes played a minor role in the redistribution of elements in the sediments.

- Geochemical classification of the sediments shows that catchment laterites are mainly ferruginous.

- Geochemical indices and REE patterns indicate that detritic sediments are mainly contributed from catchment ferruginous laterites and soils and the source rocks could have mafic characteristics.

\section{Acknowledgements}

The authors thank the members of DIPF, GELIF, DIST, LISF, LAMSF and GABAN of Vale S.A. for the field support and scientific discussions. This project was carried out in the National Forest of Carajás under permission of IBAMA (SISBIO 35594-2). Authors would like to acknowledge the financial support and field assistance provided by Instituto Tecnológico Vale (Paleoclimate Project) and CNPq (442088/2014-0). The second (JTFG) and third (PWMSF) authors were supported by CNPq through research scholarship (302839/ 2016-0 and 306450/2013-5, respectively).

\section{Appendix}

Table A1

\begin{tabular}{|c|c|c|c|c|c|}
\hline & $13 C$ & COT & $15 \mathrm{~N}$ & NT & $\mathrm{C} / \mathrm{N}$ \\
\hline LTI1-02 & -27.1 & 48.2 & 1.0 & 2.3 & 21.1 \\
\hline LTI1-03 & -26.7 & 24.2 & 1.8 & 1.7 & 13.9 \\
\hline LTI1-05 & -25.1 & 46.5 & 2.8 & 2.6 & 17.6 \\
\hline LTI1-08 & -27.1 & 27.4 & 3.2 & 1.4 & 19.0 \\
\hline LTI1-09 & -27.6 & 11.9 & 4.6 & 0.7 & 17.1 \\
\hline LTI1-12 & -29.3 & 23.1 & 3.1 & 1.7 & 13.7 \\
\hline LTI1-13 & -28.8 & 8.8 & 5.3 & 0.5 & 16.8 \\
\hline LTI1-15 & -28.0 & 7.7 & 4.8 & 0.5 & 16.7 \\
\hline LTI1-16 & -29.2 & 10.1 & 5.5 & 0.5 & 18.5 \\
\hline LTI1-17 & -28.4 & 8.6 & 4.7 & 0.5 & 18.3 \\
\hline LTI1-18 & -27.2 & 42.5 & 2.5 & 2.9 & 14.4 \\
\hline LTI1-19 & -25.4 & 44.9 & 2.2 & 2.1 & 21.3 \\
\hline LTI1-20 & -28.9 & 13.0 & 5.3 & 0.8 & 16.3 \\
\hline LTI1-21 & -29.2 & 11.9 & 5.4 & 0.6 & 19.8 \\
\hline LTI1-22 & -28.3 & 6.2 & 5.1 & 0.5 & 12.5 \\
\hline LTI1-24 & -29.0 & 9.7 & 5.3 & 0.7 & 13.5 \\
\hline LTI1-25 & -29.3 & 19.5 & 4.6 & 1.5 & 13.1 \\
\hline LTI1-26 & -29.0 & 15.5 & 4.0 & 0.8 & 19.2 \\
\hline LTI1-28 & -28.8 & 15.6 & 5.0 & 0.9 & 17.1 \\
\hline LTI1-29 & -28.7 & 8.7 & 5.3 & 0.5 & 17.5 \\
\hline LTI1-30 & -28.2 & 6.2 & 5.5 & 0.5 & 13.1 \\
\hline LTI1-31 & -28.2 & 19.9 & 4.1 & 1.4 & 14.0 \\
\hline LTI1-32 & -29.0 & 12.6 & 4.8 & 0.6 & 19.4 \\
\hline LTI1-33 & -28.4 & 46.9 & 1.5 & 2.2 & 21.7 \\
\hline LTI2-01 & -28.3 & 42.7 & 1.2 & 3.6 & 11.9 \\
\hline LTI2-02 & -27.9 & 43.1 & -0.2 & 2.8 & 15.6 \\
\hline LTI2-03 & -28.0 & 47.6 & 0.1 & 2.9 & 16.5 \\
\hline LTI2-04 & -28.6 & 52.1 & 1.8 & 3.2 & 16.3 \\
\hline LTI2-05 & -28.8 & 51.4 & 1.1 & 2.5 & 20.9 \\
\hline LTI2-06 & -28.7 & 57.1 & 0.1 & 3.3 & 17.1 \\
\hline LTI2-07 & -28.4 & 47.8 & 1.9 & 3.5 & 13.7 \\
\hline LTI2-08 & -28.3 & 52.6 & 0.8 & 2.6 & 20.3 \\
\hline LTI2-09 & -29.1 & 49.8 & 1.8 & 4.3 & 11.5 \\
\hline LTI2-11 & -27.7 & 53.1 & 1.9 & 1.6 & 32.8 \\
\hline LTI2-12 & -30.3 & 48.5 & 2.1 & 3.7 & 13.3 \\
\hline LTI2-13 & -29.6 & 52.7 & 1.8 & 4.6 & 11.4 \\
\hline LTI2-14 & -27.9 & 55.3 & 0.4 & 3.5 & 15.9 \\
\hline LTI2-15 & -28.1 & 56.9 & 1.4 & 3.4 & 16.6 \\
\hline LTI2-16 & -28.0 & 47.1 & 0.1 & 3.4 & 13.9 \\
\hline LTI2-18 & -28.1 & 51.6 & 2.4 & 3.9 & 13.2 \\
\hline LTI2-19 & -28.2 & 46.1 & 2.4 & 3.2 & 14.5 \\
\hline LTI2-20 & -29.6 & 50.8 & 1.6 & 4.3 & 11.8 \\
\hline LTI2-21 & -28.2 & 43.7 & 1.4 & 3.2 & 13.7 \\
\hline
\end{tabular}


(continued)

\begin{tabular}{|c|c|c|c|c|c|}
\hline & $13 C$ & COT & $15 N$ & NT & $\mathrm{C} / \mathrm{N}$ \\
\hline LTI2-22 & -28.4 & 53.5 & 1.6 & 2.8 & 19.3 \\
\hline LTI2-23 & -27.7 & 55.0 & 0.1 & 2.8 & 19.8 \\
\hline LTI2-24 & -27.6 & 29.0 & 2.2 & 2.1 & 14.0 \\
\hline LTI2-25 & -28.6 & 27.6 & 2.5 & 2.2 & 12.8 \\
\hline LTI2-26 & -28.3 & 50.1 & 2.2 & 3.2 & 15.4 \\
\hline LTI2-27 & -28.8 & 52.4 & 1.6 & 3.2 & 16.4 \\
\hline LTI2-28 & -27.2 & 53.2 & 1.3 & 3.6 & 14.9 \\
\hline LTI2-29 & -27.3 & 48.9 & 0.9 & 3.7 & 13.4 \\
\hline LTI2-31 & -27.3 & 53.5 & 0.9 & 3.1 & 17.5 \\
\hline LTI2-32 & -27.5 & 39.0 & 1.6 & 2.6 & 15.1 \\
\hline LTI3-02 & -25.6 & 3.1 & 6.0 & 0.3 & 12.1 \\
\hline LTI3-03 & -27.1 & 10.1 & 5.9 & 0.5 & 19.3 \\
\hline LTI3-05 & -27.1 & 15.7 & 5.9 & 0.7 & 22.3 \\
\hline LTI3-06 & -26.8 & 14.6 & 5.7 & 0.9 & 17.1 \\
\hline LTI3-10 & -27.1 & 20.6 & 4.8 & 1.5 & 14.1 \\
\hline LTI3-12 & -27.5 & 18.9 & 4.7 & 1.5 & 12.5 \\
\hline LTI3-14 & -27.5 & 17.5 & 4.5 & 1.2 & 14.2 \\
\hline LTI3-16 & -27.9 & 25.4 & 4.3 & 1.5 & 16.5 \\
\hline LTI3-17 & -27.6 & 25.0 & 4.3 & 1.4 & 18.0 \\
\hline LTI3-18 & -27.7 & 23.5 & 4.6 & 1.4 & 16.7 \\
\hline LTI3-19 & -27.6 & 20.5 & 4.6 & 1.2 & 17.4 \\
\hline LTI3-20 & -27.7 & 22.8 & 4.5 & 1.2 & 19.2 \\
\hline LTI3-21 & -27.8 & 26.9 & 4.4 & 1.6 & 17.2 \\
\hline LTI3-24 & -27.8 & 24.5 & 4.4 & 1.5 & 16.1 \\
\hline LTI3-25 & -27.0 & 21.1 & 4.2 & 1.0 & 21.5 \\
\hline LTI3-27 & -27.2 & 22.7 & 4.8 & 1.2 & 19.4 \\
\hline LTI3-28 & -27.3 & 22.4 & 4.4 & 1.0 & 21.6 \\
\hline LTI3-29 & -27.5 & 25.6 & 4.4 & 1.3 & 19.4 \\
\hline LTI3-32 & -27.0 & 29.8 & 4.2 & 1.2 & 25.5 \\
\hline LTI3-34 & -27.4 & 24.1 & 4.5 & 1.3 & 18.7 \\
\hline LTI3-35 & -27.6 & 28.2 & 4.4 & 1.4 & 20.6 \\
\hline LTI3-36 & -26.7 & 7.7 & 5.3 & 0.4 & 21.3 \\
\hline
\end{tabular}

\section{References}

Alvares, C.A., Stape, J.L., Sentelhas, P.C., Gonçalves, J.L.M., Sparovek, G., 2013 Köppen's climate classification map for Brazil. Meterologische Z. 22, 711-728.

Armstrong-Altrin, J.S., Lee, Y.I., Verma, S.P., Ramasamy, S., 2004. Geochemistry of sandstones from the upper Miocene Kudankulam Formation, southern India: implications for provenance, weathering, and tectonic setting. J. Sediment Res. $74,285-297$.

Audry, S., Grosbois, C., Bril, H., Schäfer, J., Kierczak, J., et al., 2010. Post-depositional redistribution of trace metals in reservoir sediments of a mining/smeltingimpacted watershed (the Lot River, SW France). Appl. Geochem 25 (6), $778-794$.

Babechuk, M.G., Widdowson, M., Kamber, B.S., 2014. Quantifying chemical weathering intensity and trace element release from two contrasting basalt profiles, Deccan Traps, India. Chem. Geol. 363, 56-75.

Babeesh, C., Lone, A., Achyuthan, H., 2017. Geochemistry of manasbal lake sediments, kashmir: weathering, provenance and tectonic setting. J. Geol. Soc. India 89, 563-572.

Barros, A.E., Nardi, L.V.S., Dillenburg, S.R., 2005. Geoquímica de minerais detríticos em estudos de proveniência: uma revisão. Rev. Pesqui. em Geociências 32 (1), $3-15$.

Castro, D.F., Rossetti, D.F., Pessenda, L.C.R., 2010. Quaternary paleoenvironments and relative sea-level changes in marajó island (northern Brazil): facies, $\delta 13 \mathrm{C}, \delta 15 \mathrm{~N}$ and C/N. Palaeogeogr. Palaeoclimatol. Palaeoecol. 282, 19-31.

Chen, F., Zhang, L., Yang, Y., Zhang, D., 2008. Chemical and isotopic alteration of organic matter during early diagenesis: evidence from the coastal area offshore the Pearl River estuary, south China. J. Mar. Syst. 74, 372-380.

Condie, K.C., 1991. Another look at rare earth elements in shales. Geochim. Cosmochim. Acta 55 (9), 2527-2531.

Cox, R., Lowe, D.R., Cullers, R.L., 1995. The influence of sediment recycling andbasement composition on evolution of mudrock chemistry in the southwestern United States. Geochim. Cosmochim. Acta 59 (14), 2919-2940.

Costa, M.L., Carmo, M.S., Behling, H., 2005. Mineralogia e geoquímica de sedimentos lacustres com substrato laterítico na Amazônia brasileira. Rev. Bras. Geociências 35, 165-176.

Cullers, R.L., 1995. The controls on the major and trace element evolution of shales, siltstones and sandstones of Ordovician to Tertiary age in the Wet Mountain region, Colorado, U.S.A. Chem. Geol. 123 (1-4), 107-131.

Cullers, R.L., 2000. The geochemistry of shales, siltstones and sandstones of Pennsylvian-Permian age, Colorado, USA: implications for provenance and metamorphic studies. Lithos 51, 181-203.

Das, B.K., Haake, B., 2003. Geochemistry of Rewalsar Lake sediments, Lesser Himalaya, India: implications for source area weathering, provenance and tectonic setting. J. Geosci. 7, 299-312.
Deines, P., 1980. The isotopic composition of reduced organic carbon. In: Fritz, P., Fontes, J.C. (Eds.), Handbook of Environmental Isotope Geochemistry - the Terrestrial Environment. Elsevier, Amsterdam, pp. 329-406.

Dezileau, L., Pizarro, C., Rubio, M.A., 2007. Sequential extraction of iron in marine sediments from the Chilen continental margin. Mar. Geol. 241, 111-116.

Driese, S.G., 2004. Pedogenic translocation of Fe in modern and ancient vertisols and implications for interpretations of the Hekpoort paleosol (2.25 Ga). J. Geol. $112,543-560$.

El-Leil, I.A., El-Desoky, H.M., Shafea, E.A., 2017. Geochemistry, diagenesis and mineral associations of laterite deposits along the contact zone of precambrian basement rocks and nubia sandstone between wadi dungash and wadi shait, south eastern desert. Egypt. J. Am. Sci. 13 (8), 32-54.

Fedo, C.M., Nesbitt, H.W., Young, G.M., 1995. Unravelling the effects of potassium metasomatism in sedimentary rocks and paleosols, with implications for paleoweathering conditions and provenance. Geol 23, 921-924.

Gibbs, A.K., Wirth, K.R., Hirata, W.K., Olszewski, W.J., 1986. Age and composition of the grão pará group volcanics, Serra dos Carajás. Rev. Bras.Geociênc 16 (2), 201-211.

Golder, 2010. Anexo IV e Geologia. Estudo de Impacto Ambiental, EIA Projeto Ferro Carajás S11D.

Guimarães, J.T.F., Sahoo, P.K., Souza-Filho, P.W.M., de Figueiredo, M.M.J.C., Reis, L.S., Silva, M.S., Rodrigues, T.M.R., 2016a. Holocene history of a lake filling and vegetation dynamics of the Serra Sul dos Carajás, southeast Amazonia. Na. Acad. Bras. Ciênc (in press). http://dx.doi.org/10.1590/0001-3765201720160916.

Guimarães, J.T.F., Sahoo, P.K., Souza-Filho, P.W.M., Maurity, C.W., Silva, J.R.O., Costa, F.R., Dall'agnol, R., 2016b. Late Quaternary environmental and climate changes registered in lacustrine sediments of the Serra Sul de Carajás, southeast Amazonia. J. Quatern. Sci. https://doi.org/10.1002/jqs.2839.

Hamilton, S.K., Lewis Jr., W.M., 1992. Stable carbon and nitrogen isotopes in algae and detritus from the Orinoco River floodplain, Venezuela. Geochim. Cosmochim. Acta 56, 4237-4246.

Hayashi, K., Fujisawa, H., Holland, H.D., Ohmoto, H., 1997. Geochemistry of 1.9 Ga sedimentary rocks from northeastern Labrador, Canada. Geochim. Cosmochim. Acta 61, 4115-4137.

Hermanowski, B., Costa, M.L., Carvalho, A.T., Behling, H., 2012. Palaeoenvironmental dynamics and underlying climatic changes in southeast Amazonia (Serra Sul dos Carajás, Brazil) during the late Pleistocene and Holocene. Palaeogeogr. Palaeoclimatol. Palaeoecol. 365-366, 227-246.

Herron, M.M., 1988. Geochemical classification of terrigeneous sands and shales from core or log data. J. Sediment. Petrol 58, 820-829.

Hill, I.G., Worden, R.H., Meighan, I.G., 2000. Geochemical evolution of a paleolaterite: the interbasaltic formation. North. Irel. Chem. Geol. 166, 65-84.

Horbe, A.M.C., Costa, M.L., 2005. Lateritic crust and related soils in eastern Brazilian Amazonia. Geoderma 126, 225-239.

ITV, 2016. Avaliação comparativa das características gerais das lagoas do Violão, Amendoim e Três Irmãs, Serra Sul de Carajás. ITV-DIPF (Internal report).

Jelinowska, A., Carvallo, C., Wesztergom, V., Tucholka, P., Menvielle, M., Szarka, L., Kohlbeck, F., Schott, J.J., 2000. Some magnetic properties of sediments from lake Ferto (Neusiedlersee) region, Austria-Hungary. Acta Geod. geoph. Hung 35, 255-264.

Jin, Z., Li, F., Cao, J., Wang, S., Yu, J., 2006. Geochemistry of Daihai Lake sediments, Inner Mongolia, north China: implications for provenance, sedimentary sorting, and catchment weathering. Geomorphology 80, 147-163.

Jones, B., Manning, D.A.C., 1994. Comparison of geochemical indices used for the interpretation of paleoredox conditions in ancient mudstones. Chem. Geol. 114, $111-129$.

Karunakaran, C., Sinha Roy, S., 1981. Laterite profile development linked with polycyclic geomorphic surfaces in South Kerala. In: Proceedings of the International Seminar on Lateritisation Pro- Cesses, pp. 221-231. Trivandum, India.

Lamb, A.L., Wilson, G.P., Leng, M.J., 2006. A review of coastal palaeoclimate and relative sea-level reconstructions using $\mathrm{d} 13 \mathrm{C}$ and $\mathrm{C} / \mathrm{N}$ ratios in organic material. Earth-Sci. Rev. 75, 29-57.

Latrubesse, E., 2012. Amazon lakes. In: Bengtsson, L., Herschy, R., Fairbridge, R. (Eds.), Lakes and Reservoirs. Springer Verlag, pp. 13-26.

Lelong, F., Tardy, Y., Grandin, G., Trescases, J.J., Boulange, B., 1976. Pedogenesis chemical weathering and processes of formation of some supergene ore deposits, 1976. In: Wolf, K.H. (Ed.), Handbook of Strata-bound and Stratiform Ore Deposits. Elsevier Scientific Publishing Company. Amsterdam - Printed in the Netherlands, (Chapter 3).

Macambira, M.J.B., Lafon, J.M., 1995. Geocronologia da Província mineral de Carajás: síntese dos dados e novos desafios. Boletim do Museu Paraense Emílio Goeldi. Ciências Terra 7, 263-288 (in Portuguese).

Marker, A., Friedrich, G., Carvalho, A., Melfi, A., 1991. Control of the distribution of $\mathrm{Mn}, \mathrm{Co}, \mathrm{Zn}, \mathrm{Zr}$, Ti and REEs during the evolution of laterites covers above ultramafic complexes. J. Geochem. Explor 40, 361-383.

Maurity, C.W., Kotschoubey, B., 1995. Evolução recente da cobertura de alteração no platô N1- Serra dos Carajás-PA: Degradação, pseudocarstificação, espeleotemas. Boletim do Museu Paraense Emilio Goeldi. Série Ciênc. Terra 7, 331-362.

McLennan, S.M., Nance, W.B., Taylor, S.R., 1980. Rare earth element-thorium correlations in sedimentary rocks, and the composition of the continental crust. Geochim. Cosmochim. Acta 44, 1833-1839.

McLennan, S.M., 1989. Rare earth elements in sedimentary rocks: Influence of provenance and sedimentary processes. Mineral. Soc. Amer. Rev. Mineral. 21, 169-200.

McLennan, S.M., Hemming, S., McDaniel, D.K., Hanson, G.N., 1993b. Geochemical 
approaches to sedimentation, provenance and tectonics. In: Johnsson, J.M., Basu, A. (Eds.), Processes Controlling the Composition of Clastic Sediments. Geol. Soc. Am. Spec. Pap, p. 284.

Meyers, P.A., 1994. Preservation of elemental and isotopic source identification of sedimentary organic matter. Chem. Geol. 114, 289-302.

Meyers, P.A., 1997. Organic geochemical proxies of palaeoceanographic, palaeolimnologic, and palaeoclimatic processes. Org. Geochemist. 27, 213-250.

Middelburg, J.J., Nieuwenhuize, J., 1998. Carbon and nitrogen stable isotopes in suspendedmatter and sediments from the Schelde Estuary. Mar. Chem. 60, $217-225$.

Milodowski, A.E., Zalasiewicz, J.A., 1991. Redistribution of rare earth elements during diagenesis of turbidite/hemipelagite mudrock sequences of Llandovery age from central Wales. In: Morton, A.C., Todd, S.P., Houghton, P.D. (Eds.), Developments in Sedimentary Provenance Studies, vol. 57, pp. 101-124. Geol. Soc. London Spec. Publ.

Moraes, B.C., Costa, J.M.N., Costa, A.C.L.M., Costa, M.H., 2005. Variação espacial e temporal da precipitação no estado do Pará. Acta amaz. 35 (2), 207-214.

Nesbitt, H.W., Young, G.M., 1984. Prediction of some weathering trends of plutonic and volcanic rocks based on thermodynamic and kinetic considerations. Geochim. Cosmochim. Acta 48, 1523-1534.

Nesbitt, H.W., Young, G.M., McLennan, S.M., Keays, R.R., 1996. Effects of chemical weathering and sorting on the petrogenesis of siliciclastic sediments, with implication for provenance studies. J. Geol. 104, 525-542.

Nunes, J.A., Schaefer, C.E.G.R., Ferreira Júnior, W.G., Neri, A.V., Correa, G.R., Enright, N.J., 2015. Soil-vegetation relationships on a banded ironstone 'island', Carajás Plateau, Brazilian Eastern Amazonia. An. Acad. Bras Cienc. 87, 2097-2110.

Och, L.M., Müller, B., Wichser, A., Ulrich, A., Vologina, E.G., Sturm, M., 2014, 2014 Rare earth Elem. sediments Lake Baikal. Chem. Geol. 376, 61-75.

Olszewski, W.J., Wirth, K.R., Gibbs, A.K., Gaudette, H.E., 1989. The age, origin, and tectonics of the Grão Pará Group and associated rocks, Serra dos Carajás, Brazil: Archean continental volcanism and rifting. Precambrian Res. 42, 229-254.

Pattan, J.N., Parthiban, G., Gupta, S.M., Mir, S.A., 2012. Fe speciation and Fe/Al ratio in the sediments of southeastern Arabian Sea as an indicator of climate change. Quat. Int. 250, 19-26.

Potter, P.E., Maynard, J.B., Depetris, P.J., 2005. Mud and Mudstones: Introduction and Overview. Springer, New York, p. 297.

Pourret, O., Davranche, M., 2013. Rare earth element sorption onto hydrous manganese oxide: a modeling study. J. Colloid Interface Sci. 395, 18-23.

Quinn, K.A., Byrne, R.H., Schijf, J., 2006. Sorption of yttrium and rare earth elements by amorphous ferric hydroxide: influence of $\mathrm{pH}$ and ionic strength. Mar. Chem. $99(1-4), 128-150$.

R Development Core Team, 2014. R: a Language and Environment for Statistical Computing. R Foundation for Statistical Computing, Vienna, Austria. http:// www.r-project.org.

Rämö, O.T., Dall'Agnol, R., Macambira, M.J.B., Leite, A.A.S., de Oliveira, D.C., 2002. $1.88 \mathrm{Ga}$ oxidized A-type granites of the Rio Maria region, eastern Amazonian craton, Brazil: positively anorogenic. J. Geol. 110, 603-610.

Roser, B.P., Korsch, R.J., 1988. Provenance signatures of sandstone-mudstone suites using discriminant function analysis of major-element data. Chem. Geol. 67, 119-139.

Roy, D., Roser, B.P., 2012. Geochemistry of the Tertiary sequence in the Shahbajpur-1 well, Hatia Trough, Bengal Basin, Bangladesh: Provenance, source weathering and province affinity. J. Life Earth Sci. 7, 1-13.

Sahoo, P.K., Guimarães, J.T.F., Souza-filho, P.W.M., Bozelli, R.L., et al. (in press). Limnological characteristics and planktonic diversity of five tropical upland lakes from Brazilian Amazon. Ann. Limnol - Int. J. Lim http://dx.doi.org/10. 1051/limn/2017026.

Sahoo, P.K., Guimarães, J.T.F., Souza-Filho, P.W.M., Silva, M.S., et al., 2016. Geochemistry of upland lacustrine sediments from Serra dos Carajás, Southeastern Amazon, Brazil: Implication for catchment weathering, provenance, and sedimentary processes. J. South Am. Earth Sci. 72, 178-190.

Sahoo, P.K., Souza-Filho, P.W.M., Guimarães, J.T.F., et al., 2015. A multi-proxy geochemical study of surface sediments in a plateau lake of Carajás in the southeastern Amazon region: implication for provenance and post-depositional processes. Appl. Geochem. 52, 130-146.

Sanematsu, K., Moriyama, T., Sotouky, L., Watanabe, Y., 2011. Mobility of Rare Earth Elements in Basalt-Derived Laterite at the Bolaven Plateau, Southern Laos. Resour. Geol. 61, 140-158.

Santos, P.H.C., Costa, M.L., Leite, A.S., 2016. The Piriá aluminous lateritic profile: mineralogy, geochemistry and parent rock. Braz. J. Geol. 46 (4), 617-636.

Sayyed, M.R.G., 2014. Lithological control on the mobility of elements during chemical weathering. Comun. Geol. 101 (1), 63-69.

Schellmann, W., 1981. Considerations on the definition and classification of laterites. In: Proceedings of the International Seminar on Lateritisation Processes, IGCP 129 and IAGC, Trivandrum, India. Oxford and IBH Publishing Company, New Delhi, pp. 1-10.
Schellmann, W., 1986. A new definition of laterites. Geol. Surv. India Mem. 120, 1-7. Schmitt, R.A., Smith, R.H., Lasch, J.E., Mosen, A.W., Olehy, D.A., Vasilevskis, J., 1963. Abundances of the fourteen rare-earth elements, scandium, and yttrium in meteoritic and terrestrial matter. Geochim. Cosmochim. Acta 27, 577-622.

Schnurrenberger, D., Russell, J., Kerry, K., 2003. Classification of lacustrine sediments based on sedimentary components. J. Paleolimnol. 29, 141-154.

Sifeddine, A., Martin, L., Turcq, B., Volkmer-Ribeiro, C., Soubies, F., Cordeiro, R.C. Suguio, K., 2001. Variations of the Amazonian rainforest environment: a sedimentological Record covering 30,000 years. Palaeogeogr. Palaeoclimatol. Palaeoecol. 168, 221-235.

Silva, M.S., Guimarães, J.T.F., Souza-Filho, P.W.M., Sahoo, P.K., Costa, F.R., Silva, J.R.O., Rodrigues, T.M., Costa, M.F., Submitted. Morphology and morphometry of upland lakes over lateritic crust, Serra dos Carajás, southeastern Amazon region. An. Acad. Bras. Ciênc.

Skirycz, A., Castilho, A., Chaparro, C., Carvalho, N., Tzotzos, G., Siqueira, J.O., 2014. Canga biodiversity, a matter of mining. Front. Plant Sci. 5, 1-9.

Smith, C.B., Cohen, M.C.L., Pessenda, L.C.R., França, M.C., Guimarães, J.T.F., 2012 Holocenic proxies of sedimentary organic matter and the evolution of Lake Arari-Amazon Region. Catena 90, 26-38.

Soubies, F., Suguio, K., Martin, L., Leprun, J.C., Servant, M., Turcqo, B., Fournier, M., Delaune, M., Sifeddine, A., 1991. The quaternary lacustrine deposits of the Serra dos Carajás (state of Pará, Brazil)- ages and other preliminary results. Bol. IGUSP, Publ. Esp. 8, 223-243.

Souza-Filho, P.W.M., Guimarães, J.T.F., Silva, M.S., Costa, F.R., Sahoo, P.K. Maurity, C.W., Dall'agnol, R., 2016. Basin morphology, sedimentology and seismic stratigraphy of an upland lake from Serra dos Carajás. southeastern Amazon, Brazil. Boletim do Museu Paraense Emílio Goeldi Ciências Nat. 11 (1), $71-83$.

Tapia, J., Audry, S., 2013. Control of early diagenesis processes on trace metal (Cu, $\mathrm{Zn}, \mathrm{Cd}, \mathrm{Pb}$, and $\mathrm{U}$ ) and metalloid (As, Sb) behaviors in mining and smelting impacted lacustrine environments of the Bolivian Altiplano. Appl. Geochem 31, 60-78.

Taylor, S.R., McLennan, S.M., 1985. The Continental Crust: its Composition and Evolution: an Examination of the Geological Record Preserved in Sedimentary Rocks. Oxford, U.K., Blackwell, 1985.

Teixeira, J.B.G., Eggler, D.H., 1994. Petrology, geochemistry, and tectonic setting of Archean basaltic and dioritic rocks from the N4 iron ore deposit, Serra dos Carajás, Pará, Brazil. Acta Geol. Leopold 40, 71-114.

Thornton, S.F., McManus, J., 1994. Applications of organic carbon and nitrogen stable isotope and $\mathrm{C} / \mathrm{N}$ ratios as source indicators of organic matter provenance in estuarine systems: evidence from the Tay Estuary, Scotland. Estuar. Coast. Shelf Sci. 38, 219-233.

Tribovillard, N., Algeo, T.J., Lyons, T., Riboulleau, A., 2006. Trace metals as paleoredox and paleoproductivity proxies: An update. Chem. Geol. 232, 12-32.

Turekian, K.K., Wedepohl, K.H., 1961. Distribution of the elements in some majorunits of the Earth's crust. Geol. Soc. Am. Bull. 72, 175-192.

Troxler, T.G., Richards, J.H., 2009. d13C, d15N, carbon, nitrogen and phosphorus as indicators of plant ecophysiology and organic matter pathways in Everglads deep slough, Florida, USA. Aquat. Bot. 91, 157-165.

Tyson, R.V., 1995. Sedimentary Organic Matter: Organic Facies and Palynofacies. Chapman and Hall, London.

Vermeire, M.L., Corne, S., Fekiacova, Z., Detienne, M., Delvauz, B., Cornélis, J.T., 2016 Rare Earth elements dynamics along pedogenesis in a chronosequence of podzolic soils. Chem. Geol. 446, 163-174.

Vogel, H., Wessels, M., Albrecht, C., Stich, H.B., Wagner, B., 2010. Spatial variability of recent sedimentation in Lake Ohrid (Albania/Macedonia). Biogeosciences 7, 3333-3342.

Walker, R.G., 1992. Facies, facies models and modern stratigrahic concepts. In: Walker, R.G., James, N.P. (Eds.), Facies Models - Response to Sea Level Change, second ed. Geological Association of Canada, Ontario, pp. 1-14.

Wang, S., Zhang, N., Chen, H., Li, L., Yan, W., 2014. The surface sediment types and their rare earth element characteristics from the continental shelf of the northern South China Sea. Cont. Shelf Res. 88, 185-202.

Wedepohl, K.H., 1995. The composition of the continental crust. Geochem. Cosmochim. Acta 59, 1217-1232.

Wei, Z., Jibin, X., Jixiu, C., Yanming, Z., Qiaohong, M., Jun, O., Ying, C., Zhiguo, Z Wei, L., 2010. Bulk organic carbon isotopic record of lacustrine sediments in Dahu Swamp, eastern Nanling Mountains in South China: Implication for catchment environmental and climatic changes in the last 16,000 years. J. Asian Earth Sci. 38, 162-169.

Wennrich, V., Francke, A., Dehnert, A., Juschus, O., Leipe, T., Vogt, C., brighamGrette, J., Minyuk, P.S., Melles, M., 2013. Ei'gygytgyn Scince party. Modern sedimentation process patterns in lake El'gygygyn, NE, Russia, derived from surface sediment and inlet stream samples. Clim. Past. 9, 135-148.

Xiao, S., Liu, W., Yang, S., Lai, Z., 2010. Pervasive autocorrelation of the chemical index of alteration in sedimentary profiles and its paleoenvironmental implications. Sedimentology 57, 670-676. 